\title{
Defects in Hybrid Perovskites: The Secret of Efficient Charge Transport
}

\author{
Artem Musiienko,* Davide Raffaele Ceratti, Jindřich Pipek, Mykola Brynza, \\ Hassan Elhadidy, Eduard Belas, Marián Betušiak, Geraud Delport, and Petr Praus
}

The interaction of free carriers with defects and some critical defect properties are still unclear in methylammonium lead halide perovskites (MHPs). Here, a multi-method approach is used to quantify and characterize defects in single crystal $\mathrm{MAPb}_{3}$, giving a cross-checked overview of their properties. Time of flight current waveform spectroscopy reveals the interaction of carriers with five shallow and deep defects. Photo-Hall and thermoelectric effect spectroscopy assess the defect density, cross-section, and relative (to the valence band) energy. The detailed reconstruction of free carrier relaxation through Monte Carlo simulation allows for quantifying the lifetime, mobility, and diffusion length of holes and electrons separately. Here, it is demonstrated that the dominant part of defects releases free carriers after trapping; this happens without non-radiative recombination with consequent positive effects on the photoconversion and charge transport properties. On the other hand, shallow traps decrease drift mobility sensibly. The results are the key for the optimization of the charge transport properties and defects in MHP and contribute to the research aiming to improve perovskite stability. This study paves the way for doping and defect control, enhancing the scalability of perovskite devices with large diffusion lengths and lifetimes.

the most potential to satisfy all energy demands. Photovoltaic (PV) technology effectively harvest solar energy converting it directly into electricity. Methylammonium lead halide perovskites (MHPs) are prominent PV materials thanks to their well-suited optoelectronic properties, which allowed for reaching $25.2 \%{ }^{[2]}$ of photoconversion efficiency. Furthermore, MHPs have substantial potential for other applications such as light-emitting diodes ${ }^{[3]}$ field-effect transistors, ${ }^{[4,5]}$ and photodetectors. ${ }^{[6-9]}$

Two main issues remain to be solved in MHPs: stability and defect control. Several studies probed the defect concentration, ${ }^{[10-12]}$ activation energies of traps, ${ }^{[10,11,13-18]}$ and capture cross-section ${ }^{[19]}$ in perovskites. The majority of spectroscopy methods, such as photoconductivity, photoluminescence (PL), transient spectroscopy, capacity profiling, and others, however, do not distinguish between electrons and holes; therefore they cannot

\section{Introduction}

Humankind faces unprecedented global environmental challenges mainly driven by uncontrolled greenhouse gas emissions in atmosphere. ${ }^{[1]}$ To reduce them, renewable and environmentally sustainable energy sources are necessary. Solar energy is the sustainable energy source that has

\section{A. Musiienko}

Helmholtz-Zentrum Berlin für Materialien und Energie $\mathrm{GmbH}$

Kekuléstrasse 5, 12489 Berlin, Germany

E-mail: artem.musiienko@helmholtz-berlin.de

A. Musiienko, J. Pipek, M. Brynza, H. Elhadidy, E. Belas, M. Betušiak,

P. Praus

Charles University

Faculty of Mathematics and Physics

Institute of Physics

Ke Karlovu 5, Prague 2 CZ-121 16, Czech Republic

The ORCID identification number(s) for the author(s) of this article can be found under https://doi.org/10.1002/adfm.202104467.

(C) 2021 The Authors. Advanced Functional Materials published by Wiley-VCH GmbH. This is an open access article under the terms of the Creative Commons Attribution License, which permits use, distribution and reproduction in any medium, provided the original work is properly cited. detect the exact position of the defects in the bandgap. The assignment of the nature of the defects is therefore challenging. Even if multiple studies probed lifetimes in single crystals (ranging from $10 \mu \mathrm{s}$ to $1 \mathrm{~ms}^{[20-22]}$ ) and in thin films (ranging from $100 \mathrm{~ns}$ up to a few microseconds ${ }^{[23-29]}$ ) and assessed the minority carrier diffusion length (tens of micrometers in single-crystal $\mathrm{MAPbI}_{3}{ }^{[21,22,24,30-32]}$ ) previous investigations did

DOI: 10.1002/adfm.202104467

\author{
D. R. Ceratti, G. Delport \\ CNRS \\ UMR 9006 \\ IPVF \\ Institut Photovoltaïque d'lle-de-France \\ 18 Boulevard Thomas Gobert, Palaiseau 91120, France \\ D. R. Ceratti \\ Weizmann Institute of Science \\ Department of Materials and Interfaces \\ Rehovot 7610001, Israel \\ H. Elhadidy \\ Central European Institute of Technology \\ CEITEC BUT \\ Purkynova 656/123, Brno 61200, Czech Republic
}


not provide an overall analysis of these defect and charge transport properties in the same samples. In most cases, they could not ascertain the interaction of the defects with the free charges of each sign. The dominant part of charge transport studies is performed with intense illumination which affects the occupation of traps and the lifetime of free carriers. ${ }^{[29,33]}$ In others, the phase transition ${ }^{[3-36]}$ of $\mathrm{MAPbI}_{3}$ limits the experimental range of measurement of the thermal emission of traps.

In general, determining the transport properties of electrons and holes separately is extremely challenging. To the best of our knowledge, the effect of shallow and deep defects on electrons and holes separately is not demonstrated so far. For example, the effect of non-radiative recombination on electron and hole diffusion lengths and lifetimes cannot be directly probed by time-resolved PL (TRPL). ${ }^{[37]}$ TRPL monitors the number of recombination events of free electrons with free holes. ${ }^{[38]}$ At high light fluences $\left(n=p>n_{\mathrm{t}}\right)$, the concentration of holes and electrons are similar, allowing monitoring of both carriers at the same time. On the other hand, at low light fluences $\left(n_{\mathrm{t}}>n\right)$, the recombination of the minority carriers happens with a higher probability through a trap-assisted process than by recombination with the majority carriers. Therefore the lifetime extracted from TRPL provides information on the number of minority carriers and traps only. In addition, the TRPL measurements at low light fluences are extremely difficult due to the low intensity of the light. In our ToF measurements, we are in a regime equivalent to a very low fluence (the density of minority carriers is very low, $10^{6} \mathrm{~cm}^{-3}$ ). ToF detects the probability of being trapped and detrapped for the minority carrier as it would be TRPL assuming the possibility of the detection of all recombination events through the full bulk of the sample. However, TRPL selectively describes the near-surface charge concentration (as the detection happens through an objective which focuses in a spot with a volume of $\approx 1 \mu \mathrm{m}^{3}$ ) which is dominated by surface traps (as the surface of the crystals is very different from the bulk). In addition, the diffusion of charges towards the bulk of the sample affects TRPL ${ }^{[39,40]}$ (as demonstrated in Section S6 and Figure S10, Supporting Information). It is important to notice that ToF can also identify the trapping and de-trapping process of majority carriers too. The method can indeed distinguish between electrons and holes. This is a fundamental element of our article as it provides information that is otherwise hidden in optical measurements. It also has to be noticed that TRPL lifetime is dependent on the product of the trap density and the cross-section while in ToF measurement, it is possible to distinguish between both of them.

The distinct features of electron and hole transport, which are crucial for the effective separation of free charges, remain to be properly identified. Importantly, the trap-assisted recombination of free carriers can induce chemical bond breaking ${ }^{[41]}$ and degradation of the active PV material. This paper provides the most detailed description of methylammonium lead iodide $\left(\mathrm{MAPbI}_{3}\right)$ defects existing to date, measuring their concentration, energy, capture cross-section, and charge trapping/detrapping time by several highly sensitive spectroscopy methods.

In particular, we used photo-Hall effect spectroscopy (PHES), thermoelectric effect spectroscopy (TEES), and time of flight current waveform (ToF CWF) to identify the exact position of the trap in the bandgap, and to reveal the impact of traps on electrons and holes separately. Knowing the defect parameters is the key to design defect control strategies and to understand the impact that defects play on stability. ${ }^{[3,42-47]}$ We perform our study in $\mathrm{MAPbI}_{3}$ single crystal, which describes the material itself, avoiding any influence from the substrate, grain boundaries, and any other preparation-related properties in thin films. Knowing the recombination and trapping pathways is therefore of primary importance to obtain stable MHP devices. With this paper, we elucidate the interaction of free charges with defects in MHPs and determine the artifact-free mobility and lifetime of holes and electrons.

\section{Experimental Probing and Theoretical Analysis of Charge Transfer in Single-Crystal $\mathrm{MAPbl}_{3}$}

For the first time, we used ToF CWF measurements and Monte Carlo (MC) simulations to study both electron and hole dynamics in $\mathrm{MAPbI}_{3}$. On the same samples, we use PHES and TEES to find concentration, activation energy, and capture cross-sections of traps. This allowed us to have a complete picture of the defects and their interaction with the free carriers in $\mathrm{MAPbI}_{3}$, establishing a foothold in the interpretation of other results in the literature. We visualized the electron and holes transport and their interaction with traps in a video simulation shown in Movies S1 and S2, Supporting Information. MC simulations separate the free carriers into three main groups: A) never trapped free carrier; B) detrapped carrier; and C) trapped carrier by one of the defects.

We used large $\mathrm{MAPbI}_{3}$ single crystals grown by inverse temperature crystallization (ITC) method followed by solvent evaporation. Large scale crystals (typical size of the samples is $0.6 \times$ $0.6 \times 0.2 \mathrm{~cm}^{3}$ ) are needed to obtain current waveform (CWF) measurements with good enough resolution. It would be impossible to have reliable CWF measurements on thin films due to a nonuniform electrical field. Samples were synthesized in a MAI:PbI 2 (1.1:1 mixture) solution in $\gamma$-butyrolactone, which underwent the heating procedure as shown in Figure 1a (for more details, see the methods section).

CWF measurements (Figure 1b) discern between electron and hole transport effects. We used positive and negative pulse-biases (1 ms) inducing hole and electron drift in $\mathrm{MAPbI}_{3}$, respectively. In the ToF measurements, we study charge transport in a low injection regime where the concentration of photogenerated carriers does not exceed $10^{6} \mathrm{~cm}^{-3}$. Such a low illumination regime preserves traps from significant occupation; therefore, neither electron nor hole lifetimes are affected by the filling of traps. The lifetime of electrons and holes in such a regime is affected mainly by the trapping time of deep defects. More details on the charge transport regime can be found in Section S1, Supporting Information. During the drift process, electrons and holes interact with shallow and deep traps modifying the timing of their arrival to the electrodes. From the CWF it is possible to extract the typical trapping $\left(\tau_{\mathrm{T}}\right)$ and detrapping $\left(\tau_{\mathrm{D}}\right)$ times of free carriers (more details in the Section S1 and Figure S1, Supporting Information). Shallow and deep traps induce complex non-exponential decay of the CWF (Figure 1b, which requires being analyzed accurately. 
a
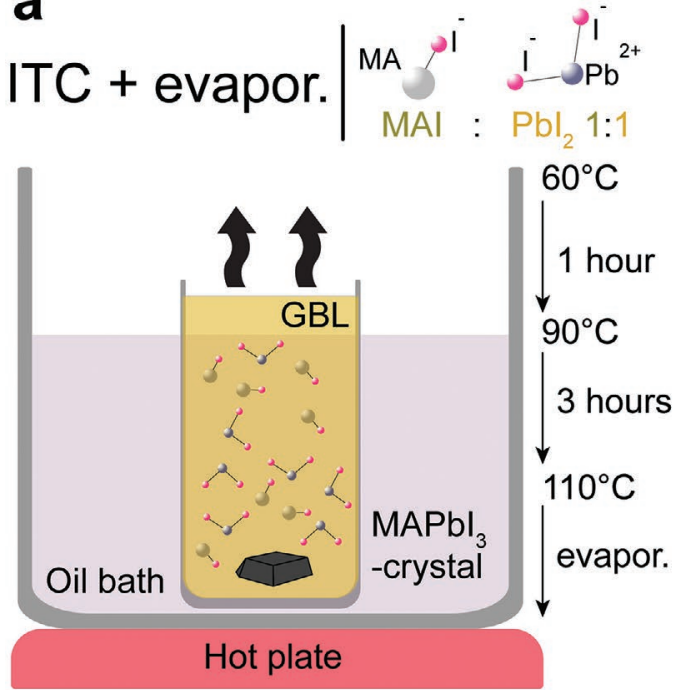

b

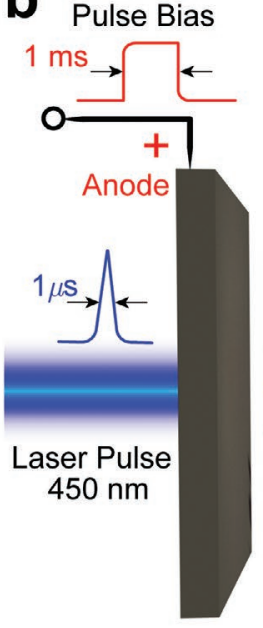

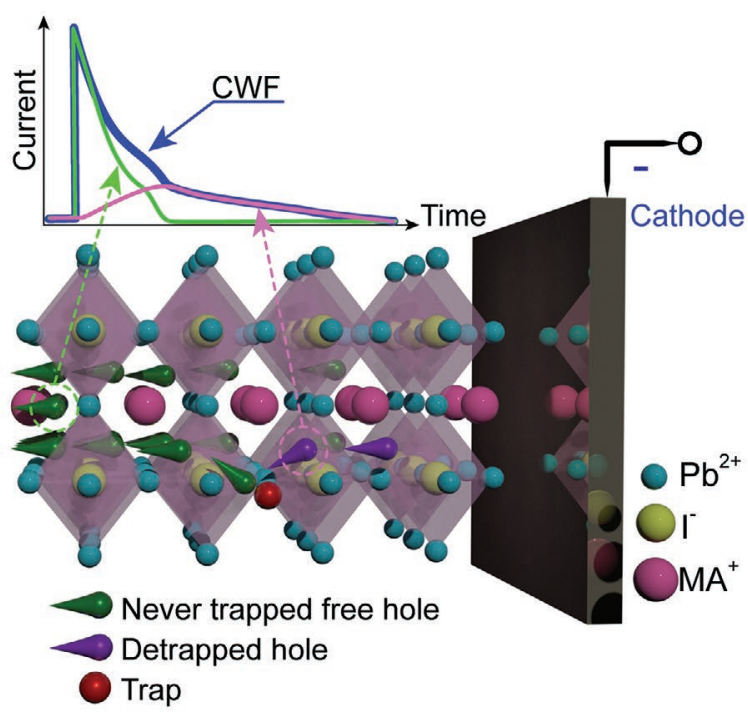

Figure 1. Perovskite growth method and schematic of ToF probing charge transport. a) Single-crystal MAPbl ${ }_{3}$ grown by ITC with slow GBL evaporation at $110^{\circ} \mathrm{C}$. b) Physical principle of ToF method. Example CWF of the ToF signal measured in $\mathrm{MAPbl}_{3}$.

\section{Effect of Traps on Charge Transport in $\mathrm{MAPbl}_{3}$}

\subsection{Free Hole Transport and Hole Traps}

Here we studied hole transport dynamics. Because the hole relaxation dynamics are different at low and high biases we changed the amplitude of the voltage pulse to have more deep insight into the interaction of traps with shallow and deep defects, as shown in Figure 2a. At $7 \mathrm{~V}$ almost all free holes fall in traps. This causes the CWF to show two typical decay times as determined by a faster decay in the time range of 1-10 $\mu \mathrm{s}$, due to holes falling in shallow traps, followed by a slower decay in the range of 10-45 $\mu$ s due to holes falling in deep traps. At higher voltage bias, $30 \mathrm{~V}$, a large part of the holes drifts to the electrode without falling into deep traps. This causes the CWF to have third decay presumably produced by the arrival of delayed holes to the electrode, starting at transit time, $T_{\mathrm{R}}=52 \mu \mathrm{s}$, the time needed for the free holes to reach the electrode. The non-exponential shape of CWF reflects the presence of shallow and deep hole traps in the $\mathrm{MAPI}_{3} \cdot{ }^{[48]}$

Using MC simulations we analyze the evolution of the hole cloud and trap-occupation at $t=T_{\mathrm{R}}$ shown in Figure 2b,d. The hole-CWF can be fitted by an MC simulation having one shallow $\left(E_{1}\right)$ and one deep trap $\left(E_{2}\right)$. Hereinafter, we number defects according to their energy in respect to the valance band. The shallow trap $E_{1}$ is responsible for fast trapping (trapping time $\tau_{\mathrm{T} 1}=3.5 \mu \mathrm{s}$ ) and fast detrapping (detrapping time $\tau_{\mathrm{D} 1}=$ $4 \mu \mathrm{s}$ ) of free holes. Figure $2 \mathrm{c}$ in yellow, shows the occupancy of the shallow trap, which increases rapidly after the laser pulse, peaks at $4 \mu \mathrm{s}$, and decreases more slowly. The trapping-detrapping phenomenon of the shallow trap $E_{1}$ delays the hole cloud drift, which translates to reduced hole mobility. A second effect of the shallow trap is the broadening of the charge cloud distribution in space, as demonstrated in Figure $2 \mathrm{~b}, \mathrm{~d}$ by never trapped and detrapped holes.
Contrary to the shallow trap $E_{1}$, the deep trap $E_{2}$ permanently traps free holes (as shown in Figure $2 \mathrm{~b}, \mathrm{~d}$ ) with $\tau_{\mathrm{T} 2}=60 \mu \mathrm{s}$ and $\tau_{\mathrm{D} 2} \gg 20 \mathrm{~ms}$ (temporal limit of our instrument). Traps fill with a nonlinear spatial profile $\left(p_{\mathrm{t} 2}(x)\right)$ in $\mathrm{MAPbI}_{3}$. Figure $2 \mathrm{c}$ shows the temporal occupancy of the deep hole trap $E_{2}\left(p_{\mathrm{t} 2}(t)\right)$. It slowly increases when the hole cloud moves through the bulk toward the cathode and never decreases since the holes are not released. The concentration of deep trapped holes remains constant after $T_{R}$ since no more holes are available after the cloud reached the cathode. According to ToF and MC results, deep trap $E_{2}$ is the only hole recombination path in $\mathrm{MAPbI}_{3}$. Thus, the lifetime of the free hole $\left(\tau_{\mathrm{h}}\right)$ is equal to the trapping time of $E_{2}, \tau_{\mathrm{T} 2}$, which gives $\tau_{\mathrm{h}}=\tau_{\mathrm{T} 2}=60 \mu \mathrm{s}$

MC simulations distinguished the component of the CWF due to never-trapped and trap-delayed holes, Figure 2a. The transit time of (never trapped) free holes defines a "pure" hole drift mobility $\left(\mu_{\mathrm{h}}\right)$ of $21 \mathrm{~cm}^{2} \mathrm{~V}^{-1} \mathrm{~s}^{-1}$ (as extracted by Figure S2a, Supporting Information) which, combined with the hole lifetime $(60 \mu \mathrm{s})$ provides ${ }^{[49]}$ a very elevated hole diffusion length $\left(L_{\mathrm{h}}\right)$ of $56 \mu \mathrm{m}$, and a diffusion coefficient $\left(D_{\mathrm{h}}\right)$ of $0.53 \mathrm{~cm}^{2} \mathrm{~s}^{-1}$. The hole mobility in $\mathrm{MAPbI}_{3}$ is larger than in $\operatorname{MAPbBr}_{3}\left(12 \mathrm{~cm}^{2} \mathrm{~V}^{-1} \mathrm{~s}^{-1}\right.$ as we measured $\left.{ }^{[48]}\right)$ due the polaronic nature of mobility (or strong hole-phonon coupling). Polarons are affected by the lead-halide bond strength where the $\mathrm{Pb}-\mathrm{Br}$ bond is more ionic than the $\mathrm{Pb}-\mathrm{I}$ bond. ${ }^{[50]}$ Finalizing analysis of the free hole transport, we also estimate the hole surface recombination pathway with a typical velocity (SRV or $\left.S_{\mathrm{h}}\right) S_{\mathrm{h}}<$ $500 \mathrm{~cm} \mathrm{~s}^{-1}$ found from the ToF bias dependence [Section S2 and Figure S2, Supporting Information]. Classical semiconductors such as silicon show SRV over $1000 \mathrm{~cm} \mathrm{~s}^{-1}$. ${ }^{[51]}$ We relate slow surface recombination velocity-proportional to the concentration of defects on the surface-to the self-healing ${ }^{[52-56]}$ nature of $\mathrm{MAPI}_{3}$. MAPbI 3 shows weak structural bonds $(\mathrm{Pb}-\mathrm{I})$ that can be considered to continuously brake and reform. ${ }^{[57]}$ Because of this, the kinetic re-formation of a damaged surface similar to the known phenomenon of Ostwald ripening 

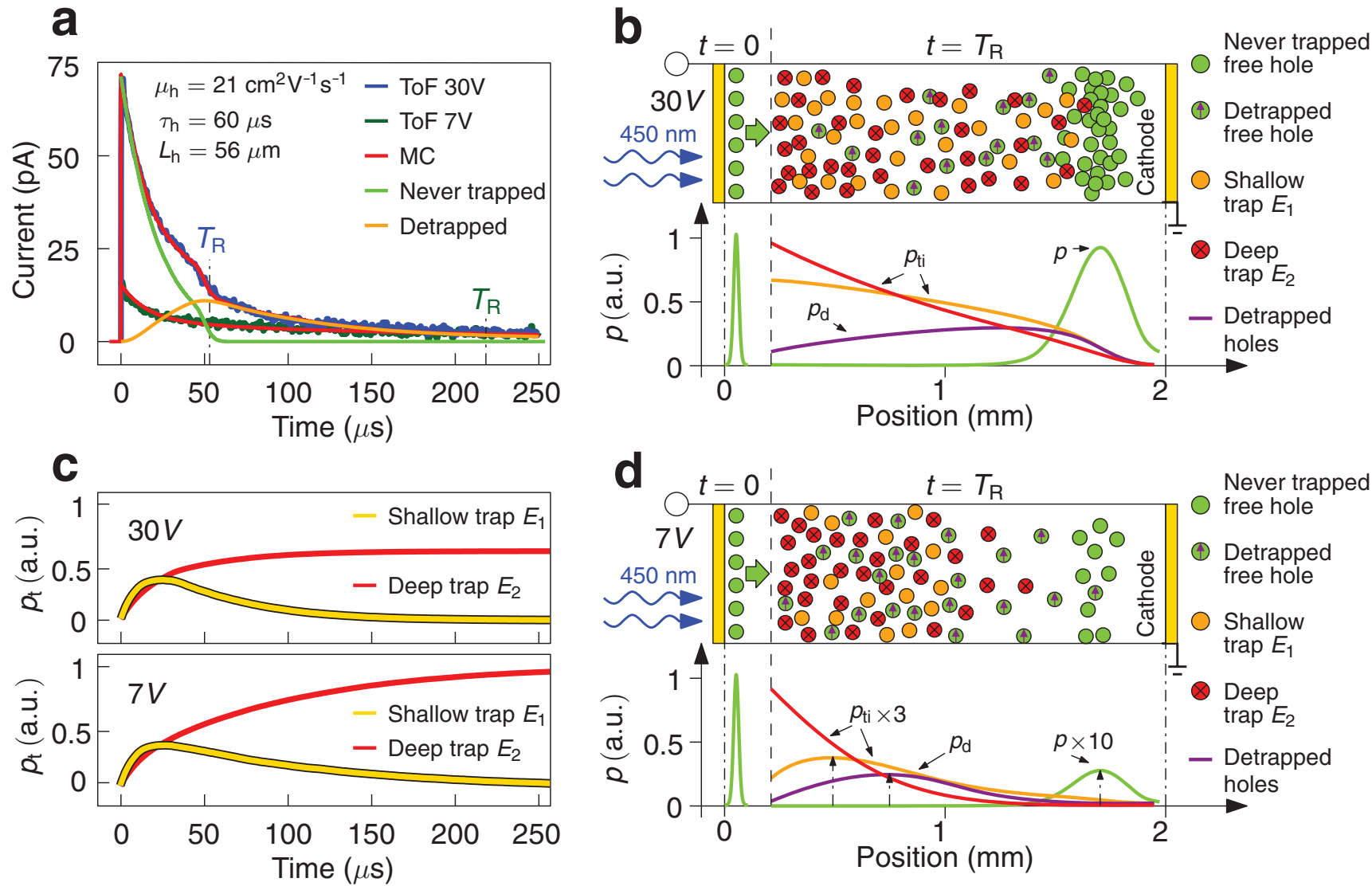

Figure 2. Hole transport properties and hole-defect interaction. a) ToF CWF spectroscopy and MC simulation of hole transport at 30 and $7 \mathrm{~V}$ biases. b,d) Simulation of the hole cloud drifting in the bulk of $\mathrm{MAPbl}_{3}$ affected by shallow and deep hole traps at 30 and $7 \mathrm{~V}$ biases, respectively. The quantitative spatial distribution of trapped holes is shown at the bottom. $t=0$ corresponds to the hole distribution after light pulse absorption. $t=T_{\mathrm{R}}$ represents the evolving hole cloud when it reaches the vicinity of the cathode on the right side. c) Temporal evolution of trap occupation at 30 and $7 \mathrm{~V}$ biases.

takes place in short times, amending many of the defects that would be otherwise present on other, harder, semiconductor surfaces. Similar effects of self-healing ${ }^{[52-56]}$ were observed by Yadavalli et al. ${ }^{[57]}$ which noted cracks formed in mechanically stretched thin films disappear over time once the tensile stress is removed. This effect is relevant when the surfaces are macroscopically smooth and have scratches below $1 \mu \mathrm{m}$ as it is in our case, thanks to our accurate mechanical polishing procedure (see the Experimental Section).

To clarify the hole-transport dynamics we report the Movie S1, Supporting Information, which shows the time and space evolution of the hole cloud in $\mathrm{MAPbI}_{3}$ at 30 and $7 \mathrm{~V}$ biases. The video shows free holes, detrapped holes, and trapped holes by shallow and deep traps. The movies cover a broad range of holes dynamics ranging from $50 \mathrm{~ns}$, where light generates free holes up to $300 \mu$ s where the cathode collects all holes. More details can be found in Section S4, Supporting Information.

\subsection{Free Electron Transport and Electron Traps}

We performed a very similar analysis on electron drift by applying negative biases of -30 and $-70 \mathrm{~V}$ required to detect clear signals. We obtained the value of the "pure" electron mobility of $4.0 \mathrm{~cm}^{2} \mathrm{~V}^{-1} \mathrm{~s}^{-1}$ (Figure 3a and Figure S2b, Supporting Information) five times smaller than for holes. Electron CWFs show a fast decay in the range of $2 \mathrm{~ns}-3 \mu \mathrm{s}$ followed by a slow decay in the range of 3-120 $\mathrm{s}$. MC simulations indicate that the electron transport is influenced by deep trap $\left(E_{3}\right)$ with trapping time $\tau_{\mathrm{T} 3}=120 \mu \mathrm{s}$ and $\tau_{\mathrm{D} 3} \gg 20 \mathrm{~ms}$ and two shallow traps $\left(E_{4}\right.$ and $\left.E_{5}\right)$ as shown in Figure $3 \mathrm{~b}, \mathrm{~d}$. Also in this case the electron lifetime $\left(\tau_{\mathrm{e}}\right)$ is equal to the trapping time of the only deep trap $E_{3}$. We have therefore $\tau_{\mathrm{e}}=\tau_{\mathrm{T} 3}=120 \mu \mathrm{s}$. Among all samples (eight samples), we observed deviations in electron lifetime up to $26 \mu \mathrm{s}$, as shown in Figure S4c,d, Supporting Information. In the case of holes, we observe values between 60 and $200 \mu \mathrm{s}$ (Figure S4a,b, Supporting Information). We did not observe the variation of mobility larger than $12 \%$. Electron and hole lifetimes can vary between samples implying that the crystal growth conditions can be slightly different between each crystal in the function of the time at which the first nuclei appeared. These may modify the defect densities in the perovskite and, therefore, their transport properties. A detailed study of the influence of the growth conditions on the defect properties in $\mathrm{MAPbI}_{3}$ is beyond the scope of this research.

The shallow electron traps $E_{4}$ (with $\tau_{\mathrm{T} 4}=100 \mu$ s and $\tau_{\mathrm{D} 4}=$ $100 \mu \mathrm{s}$ ) and $E_{5}$ (with $\tau_{\mathrm{T} 5}=2.5 \mu \mathrm{s}$ and $\tau_{\mathrm{D} 5}=1.5 \mu \mathrm{s}$ ) are responsible 

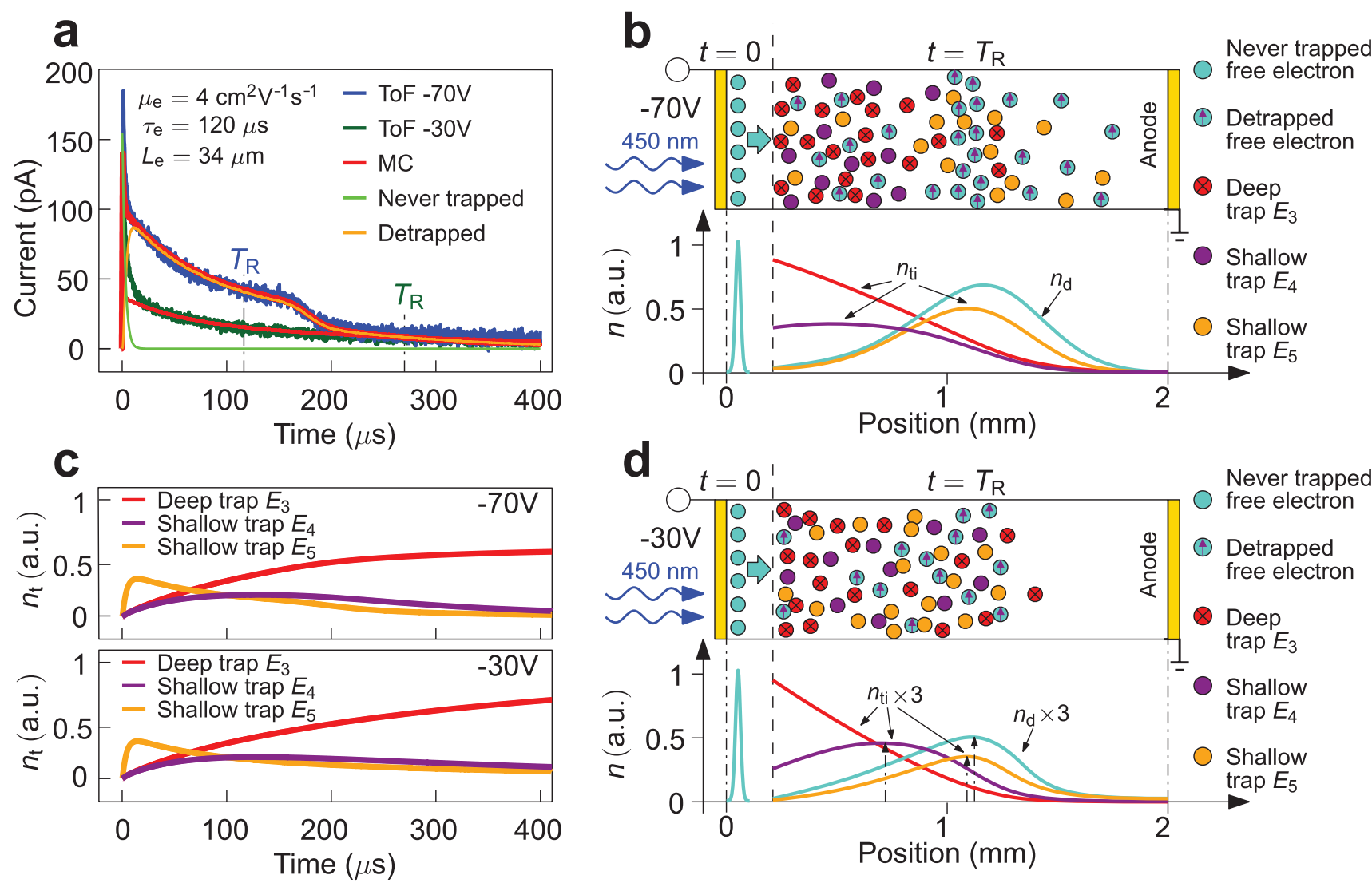

Figure 3. Electron transport properties and electron-defect interaction. a) ToF spectroscopy and MC simulation of electron transport at -70 and $-30 \mathrm{~V}$ biases. b, d) Simulation of the electron cloud drifting in the bulk of $\mathrm{MAPbl}_{3}$ affected by shallow and deep electron traps at -70 and $-30 \mathrm{~V}$ biases, respectively. c) Temporal evolution of trap occupation at -70 and $-30 \mathrm{~V}$ biases.

for the fast trapping and fast detrapping of electrons. Quick trapping time rapidly occupies the shallow trap $E_{5}$ at all biases, as shown in Figure 3c The occupation of the trap peaks at $t=$ $2 \mu \mathrm{s}$. The shallow electron trap, $E_{4}$, occupancy follows a similar trend but on $100 \mu$ s timescale due to longer trapping-detrapping times. Movie S2, Supporting Information, (-70 and $-30 \mathrm{~V})$ shows the whole process of the time and space evolution of the electron cloud in $\mathrm{MAPbI}_{3}$. Slow electron SRV $S_{\mathrm{e}}<100 \mathrm{~cm} \mathrm{~s}^{-1}$ was estimated from the ToF bias dependence (Section S2 and Figure S2e,f, Supporting Information).

\subsection{Delaying Effect of Traps on Holes and Electrons}

We have previously extracted the "pure" electron and hole mobilities; however, a shallow hole trap $\left(E_{1}\right)$ and electron traps $\left(E_{4}\right.$ and $\left.E_{5}\right)$ delay the electrons and holes during the charge separation process. Therefore the center of the electron and hole clouds advances more slowly than the never-trapped carriers. Through MC simulations, we can track the trapped and detrapped carriers (Figures $3 \mathrm{~b} d$ and $2 \mathrm{~b}, \mathrm{~d}$ ) and calculate an effective electron $\left(\tilde{\mu}_{\mathrm{e}}\right)$ and hole $\left(\tilde{\mu}_{\mathrm{h}}\right)$ mobilities, as shown in Figure $4 \tilde{\mu}_{\mathrm{e}}$ and $\widetilde{\mu}_{\mathrm{h}}$ decrease to the values of 1.7 and $10 \mathrm{~cm}^{2} \mathrm{~V}^{-1} \mathrm{~s}^{-1}$, respectively. One should notice that the $\tilde{\mu}_{\mathrm{e}}(E)$ has more than one inflection due to the presence of two shallow traps $E_{4}$ and $E_{5}$ (cf. Figure $4 a$ ). The extreme values of effective electron and hole mobilities are in complete agreement with effective steady-state mobilities $\left(\tilde{\mu}_{\mathrm{eff}}^{E 4+E 5}\right.$ and $\left.\tilde{\mu}_{\mathrm{eff}}^{E 1}\right)$ found by taking into account all shallow electron traps (as shown by green dashed lines in Figure 4a,b):

$$
\tilde{\mu}_{\mathrm{eff}}^{E 4+E 5}=\mu_{\mathrm{e}} \frac{1}{1+\frac{\tau_{D 4}}{\tau_{T 4}}+\frac{\tau_{D 5}}{\tau_{T 5}}}
$$

Free hole drift maintains a Gaussian profile (Figure 2b,d) and non-dispersive transport (for more details, see Section S2, Supporting Information). The Gaussian distribution indicates the Fröhlich large polaron (or strong hole-phonon coupling) rather than small polaron hopping assisted transport. ${ }^{[58]}$ In principle, the treatment of CWF at low biases by dispersing power-law relation (in log-log scale) leads to notable mistreatment of experimental data, transit time, and, as a result, mobility value (Figure S3, Supporting Information). For example, the dispersive law gives an overestimated mobility of $64 \mathrm{~cm}^{2} \mathrm{~V}^{-1} \mathrm{~s}^{-1}$ at $7 \mathrm{~V}$, while the $\mathrm{MC}$ simulation gives a correct description of the hole drift and mobility $\left(21 \mathrm{~cm}^{2} \mathrm{~V}^{-1} \mathrm{~s}^{-1}\right)$ at all biases. The drift mobility found in this study is in agreement with electron $\left(0.7-1.4 \mathrm{~cm}^{2} \mathrm{~V}^{-1} \mathrm{~s}^{-1}\right)^{[59,60]}$ and hole $\left(20-35 \mathrm{~cm}^{2} \mathrm{~V}^{-1} \mathrm{~s}^{-1}\right)^{[30,61-64]}$ mobilities found in the literature. The lower electron mobility values reported in other studies can be explained by the effect of shallow traps on electron transport, also shown in this study. One should note that the larger mobility values of over $64 \mathrm{~cm}^{2} \mathrm{~V}^{-1} \mathrm{~s}^{-1}$ 
2

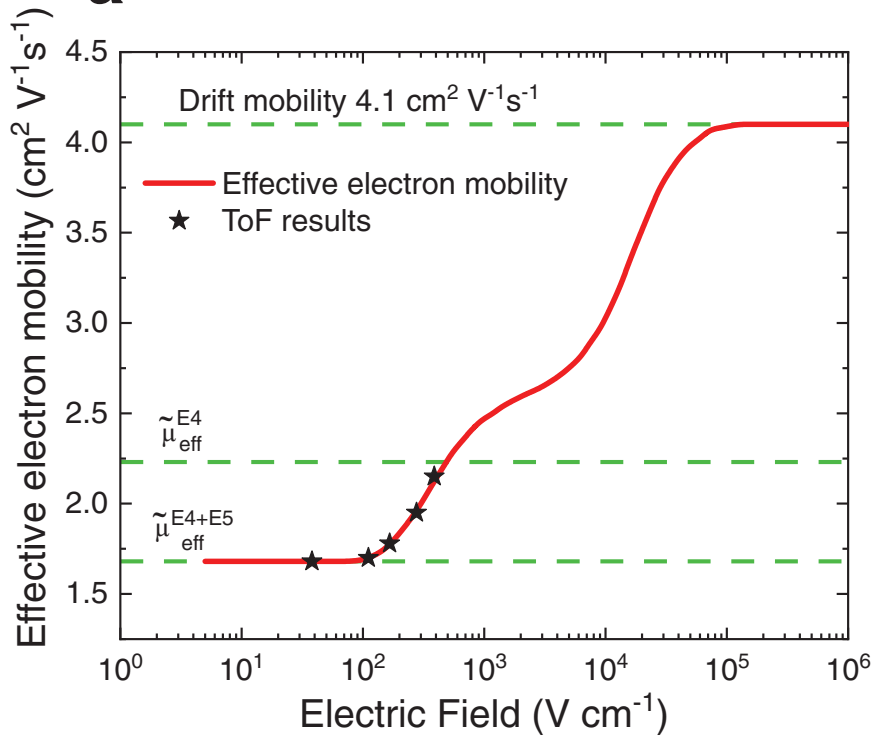

b

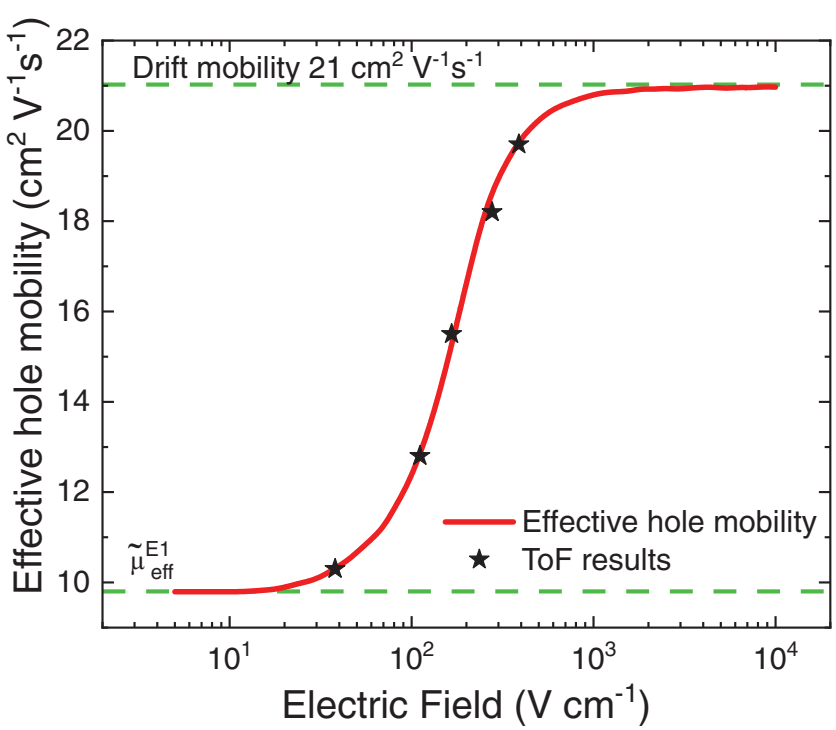

Figure 4. Delaying of electrons and holes by shallow traps. a) The electron effective mobility affected by shallow electron traps $E_{4}$ and $E_{5}$ as a function of the electric field. b) The effective hole mobility affected by shallow hole trap $E_{1}$ as a function of the electric field.

are reported in articles that use simplified analytical equations which allow for evaluating only the order of magnitude of the mobility. ${ }^{[31,65,66]}$ The activity of traps should be taken in other characterization methods such Hall effect, ${ }^{[29,67]}$ terahertz conductivity probes, ${ }^{[68,69]}$ time of flight, and others.

\section{Traps Energetics and Parameters}

\subsection{Photoactivation of Traps in a Magnetic Field}

ToF identifies the effect of traps on charge transport, but it does not allow us to determine trap concentration or activation energy. For this reason, we performed PHES and TEES to complete our view on the fingerprints of defects in $\mathrm{MAPbI}_{3}$. We mark PHES energy as $E_{\mathrm{H}}$ and TEES energy as $E_{\mathrm{T}}$. Figure 5a reports the photoconductivity $(\mathrm{PhC})$ spectrum in the $0.5-1.8 \mathrm{eV}$ range, which we fitted according to the photoionization cross-section: ${ }^{[70]}$

$\sigma_{h v}=\frac{C\left(h v-E_{\mathrm{t}}\right)^{1.5}}{h v\left(h v-E_{\mathrm{t}}\left(1-m / m_{\mathrm{ef}}\right)\right)^{2}}$

Where $h v, E_{\mathrm{t}}, C$, and $m_{\mathrm{ef}}$ are the photon energy, photoionization energy of trap, constant, and effective hole mass. We observe an increase of PhC starting from $0.6 \mathrm{eV}$ associated with the optical excitation of charge carriers from the deep trap $E_{2}$ followed by the second ionization of trap $E_{3}$ at $0.75 \mathrm{eV}$. The two shallower traps were identified at 1.2 and $1.35 \mathrm{eV}$. The peak of absorption was observed at $1.55 \mathrm{eV}$, which is the edge of the band-to-band transition. The PhC spectroscopy is a highly sensitive technique allowing us to resolve a low concentration of defects. On the other hand, $\mathrm{PhC}$ cannot distinguish electrons from holes and cannot find this way how activation energies are linked to the valence or conduction bands. One should also notice that shallow traps $E_{4}$ and $E_{5}$ mix their $\mathrm{PhC}$ signals for $h v>1.25 \mathrm{eV}$ because of continuous interaction with the valence and conduction bands and resolution of a probed light of $0.1 \mathrm{eV}$.

By photo-Hall measurements (Figure $5 \mathrm{~b}$ ) we determined the relative energy between the defects. We observed an increase of the Hall mobility with a positive Hall voltage (Figure S5, Supporting Information) in the energy regions $0.6-0.75,0.8-1.1$, and $1.2-1.4 \mathrm{eV}$, indicating the transition of the electrons from the valance band to the defects. Such transition leads to free hole generation in the valance band. Thus, we assign the position of deep traps found by PHES as $E_{\mathrm{H} 2}=E_{\mathrm{v}}+0.6 \mathrm{eV}$ and $E_{\mathrm{H} 3}=E_{\mathrm{v}}+0.75 \mathrm{eV}$ as shown in Figure 6b. Concerning shallow traps, one should notice that their assignment by PHES is challenging. Shallow traps can be activated by a two-step photoninduced ionization and subsequent thermal filling generation process ${ }^{[1]}$ which decreases the energy sensitivity of the measurement.

\subsection{Thermal Activation of Traps}

To identify the thermal activation energies, concentration, and capture cross-section of defects, we used TEES. Defects typically show slightly different optical and thermal ionization energies. TEES allows determining the position of a defect in the bandgap and its thermal ionization energy. We studied the thermal emission current as a function of temperature in the range of $300-340 \mathrm{~K}$ using different heating rates $0.6-1.0 \mathrm{~K} \mathrm{~min}^{-1}$. Figure $5 \mathrm{c}$ shows TEES bands obtained below the phase transition temperature $^{[75]}$ which should also allow excluding heat induces lattice defects (formed for $T>350 \mathrm{~K}$ ). TEES bands showed activation energies of 1.0 and $1.25 \mathrm{eV}$ as well as electron capture crosssections $5 \times 10^{-13}$ and $2 \times 10^{-11} \mathrm{~cm}^{2}$, respectively fitted by the 

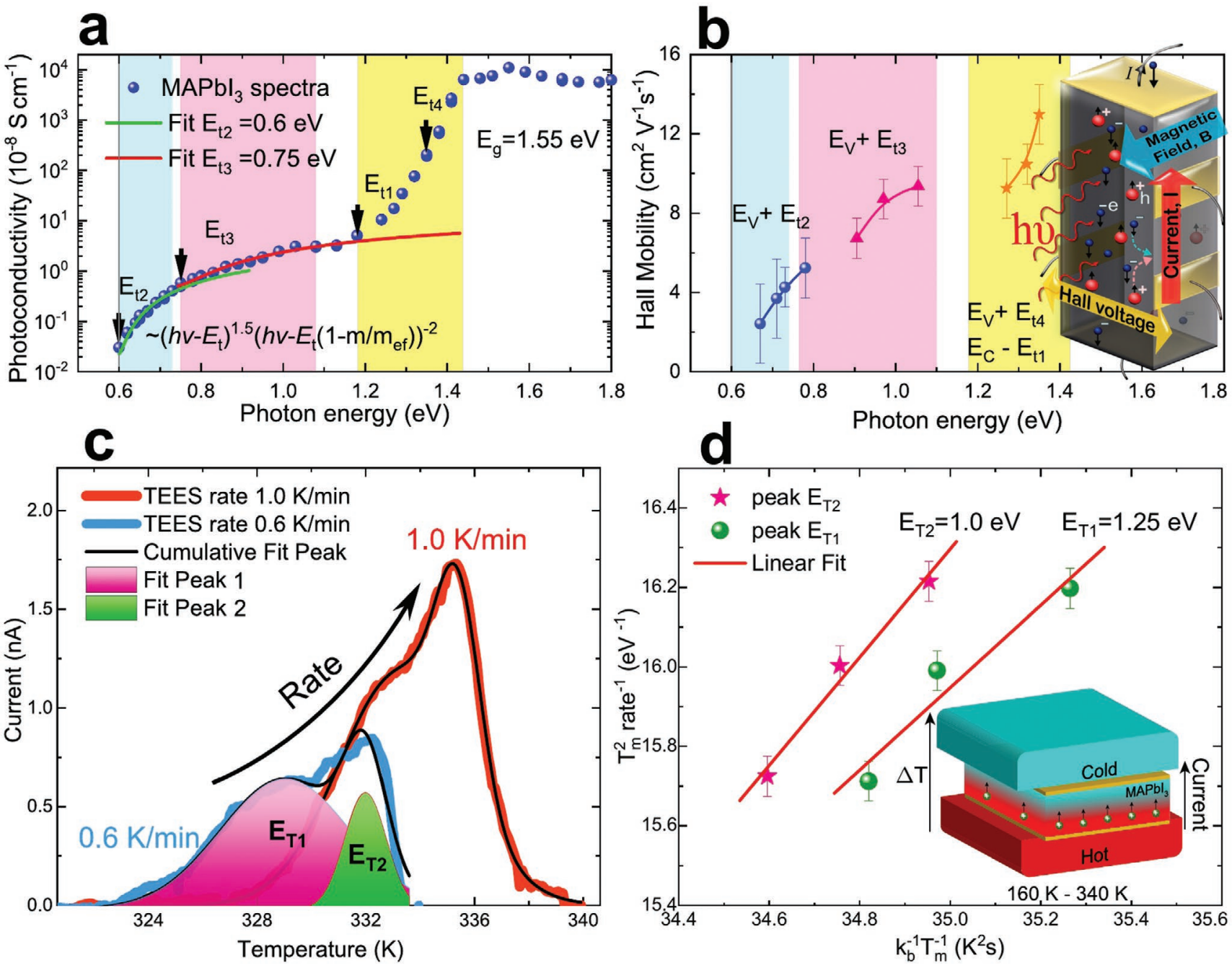

Figure 5. Revealing fingerprints of defects in $\mathrm{MAPbl}_{3}$. a) Photoconductivity spectrum in the photon energy range of $0.5-1.8 \mathrm{eV}$. Fitting curves represent trap-to-band transitions according to the analytical solution of optical capture cross-section. ${ }^{[70]}$ Vertical arrows show threshold energies. b) Hall mobility spectrum showing free hole signal excited by the light. The inset represents the basic physical principles of the PHES method. c) TEES signal in the range of 300-340 K measured at different heating rates. The Gaussian band shows the fitting of the TEES signal. d) Arrhenius plot of the peak temperatures. The inset demonstrates the basic physical principles of the TEES method.

Arrhenius plot in Figure $5 \mathrm{~d}^{[76]}$ We obtained defect concentrations of $N_{\mathrm{t} 2}=10^{10} \mathrm{~cm}^{-3}$ and $N_{\mathrm{t} 1}=5.0 \times 10^{10} \mathrm{~cm}^{-3}$ by integrating the bands in Figure $5 \mathrm{c}$. The positive sign of the current allows assigning the relative position of the traps to $E_{\mathrm{T} 2}=E_{\mathrm{C}}-1.0$ and $E_{\mathrm{T} 1}=E_{\mathrm{C}}-1.25 \mathrm{eV}$, which are in good agreement with PHES ionization energies, as shown in Figure 6b.

To resume, using the results from time-resolved ToF, steady-state PHES, and TEES, we could also estimate the hole and electron capture cross-section of the defects. (Table 1 and Figure 6). Trap $E_{1}$ is, as revealed by all methods, a shallow hole trap near the valence band with a hole capture cross-section of $10^{-13} \mathrm{~cm}^{2}$. $E_{1}$ reduces the hole mobility, as was shown by ToF. $E_{1}$ also has a large electron capture cross-section, and could shorten the free carries lifetime in high photo-injection regimes (trap filling of $E_{2}$ ) considering the slightly p-type doped nature of the bulk, demonstrated by possible PHES. Trap $E_{2}$, observed by all methods, is identified as a deep hole trap and has a hole capture cross-section of $\approx 10^{-14} \mathrm{~cm}^{2}$. This trap controls the lifetime of free holes, as directly shown by ToF. Defect $E_{2}$ has a large electron capture cross-section, which makes it responsible for free-charge recombination. TEES revealed the concentration of hole traps below $10^{11} \mathrm{~cm}^{-3}$. The concentration of deep defects is consistent with previous studies $^{[10,21,77-80]}$ which suggests that the other information deduced by the technique is also correct.

The shallow electron traps concentration $\left(E_{4}\right.$ and $\left.E_{5}\right)$ can be estimated roughly at $10^{12} \mathrm{~cm}^{-3}$ by using PHES activation energies and ToF trapping/detrapping times (more details in Section S1, Supporting Information). Similarly, we found electron capture cross-sections of $10^{-15}$ and $10^{-14} \mathrm{~cm}^{2}$ for $E_{4}$ and $E_{5}$, respectively. These defects dramatically decrease electron effective mobility. Deep trap $E_{3}$, as observed by ToF and PHES, is a deep electron trap with a capture cross-section of $10^{-14} \mathrm{~cm}^{2}$ and a concentration of $10^{11} \mathrm{~cm}^{-3}$, limiting the lifetime of the 

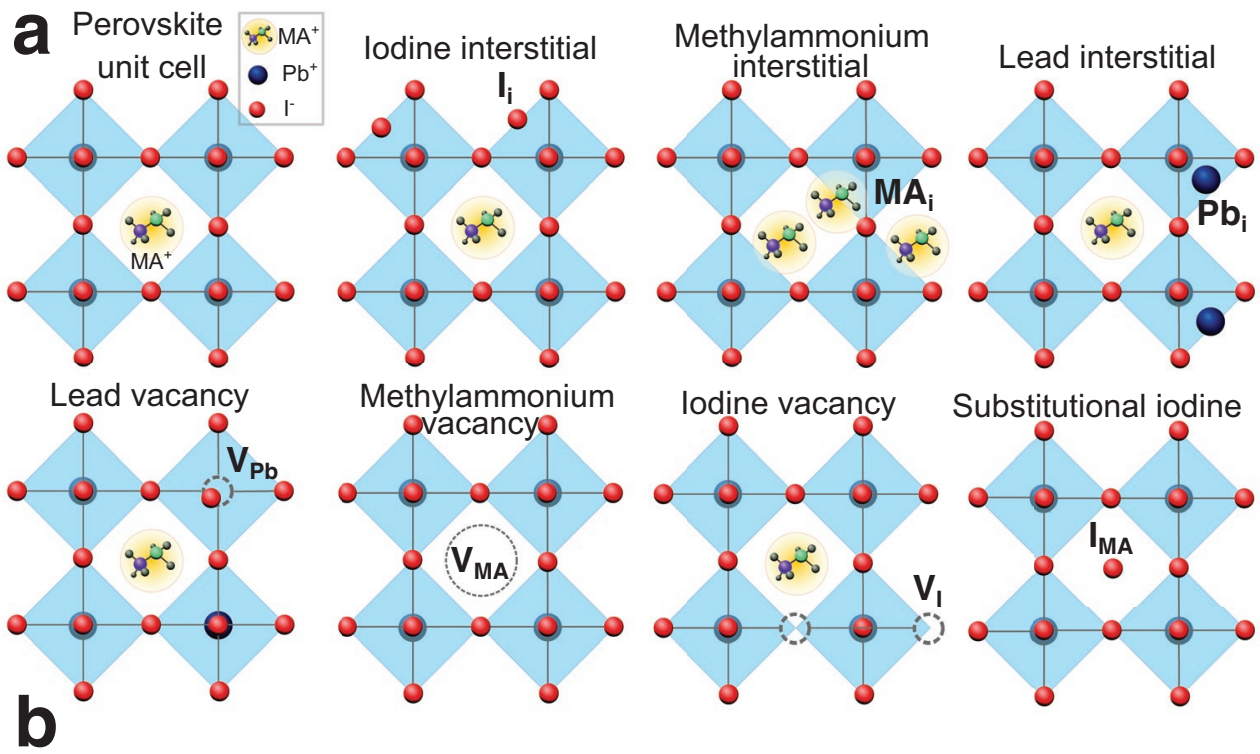

Substitutional iodine
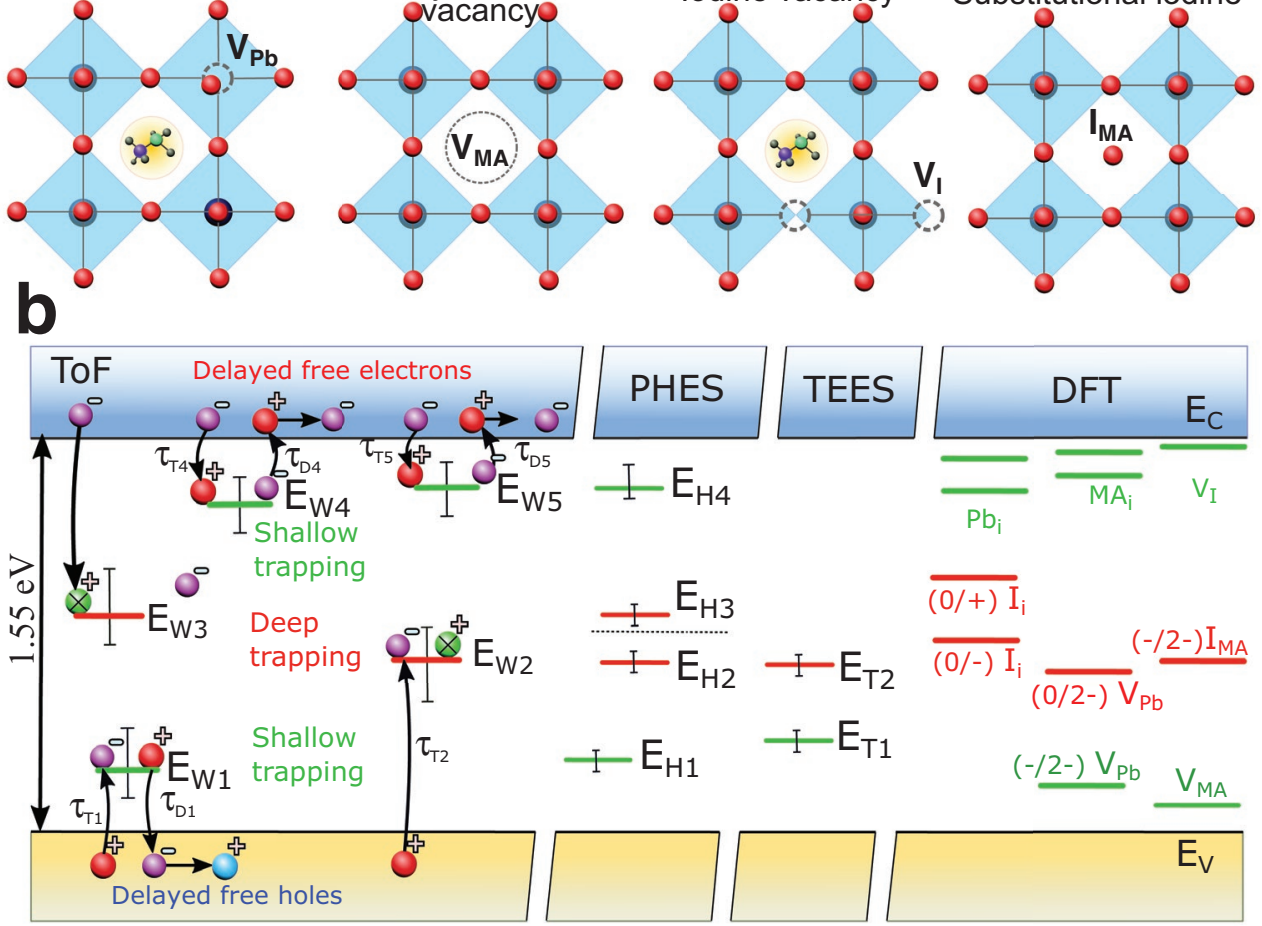

$\overline{(0 /+) I_{i}}$
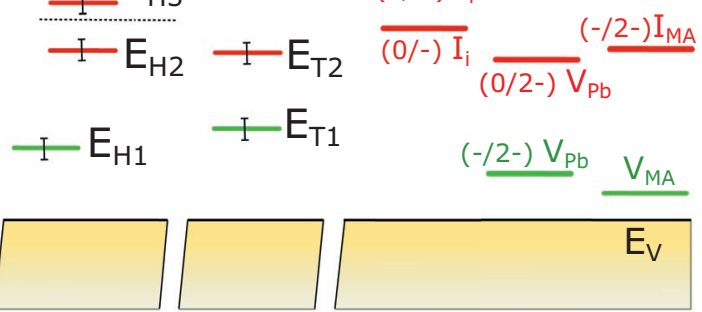

Figure 6. Energetic distribution of defects and their chemistry in $\mathrm{MAPbl}_{3}$. a) Defects in $\mathrm{MAPbl}_{3}$. b) Experimentally estimated trap activation energies according to ToF, PHES, and TEES in this study. Possible origin of traps according to the first principle DFT simulations in other studies. ${ }^{\text {72-74] }}$

electron. The trap is located near Fermi level (roughly $E_{\mathrm{g}} / 2=$ $0.77 \mathrm{eV}$ ) which explains why it could not be measured by TEES.

\subsection{Ion Migration}

In this study, we do not observe the effect of the ion migration on the measurements due to the very low ion mobility and the long transit time of mobile ions which reaches $1000 \mathrm{~s}^{[48,81-87]}$ in single-crystals with thicknesses of $2 \mathrm{~mm}$ (corresponding to the electrical field of $300 \mathrm{~V} \mathrm{~cm}^{-1}$ ). The contribution of ions in ToF CWFs is also negligible, since the maximum transit time for free electrons is $150 \mu \mathrm{s}$. In principle, ion migration can cause ion accumulation and electrical field deformation. We do not observe electrical field deformation for the hole or electron CWF. This is proved by the linear trend between carrier transit time and electrical field applied to the sample (Figure S2a,b, Supporting Information). Nevertheless, to prevent possible accumulation of ions we use short bias pulses $(1 \mathrm{~ms})$ in ToF measurements. Duijnstee et al. demonstrated that such short pulses do not lead to perturbation of ions in halide perovskite. ${ }^{[87]}$

\subsection{Unraveling the Defect Nature}

After measuring and identifying the energy, density, and other parameters of each defect, it is noteworthy to assign a chemical identity to each of them. Density functional theory (DFT) predictions identified methylammonium interstitial $\left(M A_{\mathrm{i}}\right)$, iodine interstitial $\left(I_{\mathrm{i}}\right)$, and lead vacancy $\left(V_{\mathrm{Pb}}\right)$ defects as the most stable defects ${ }^{[1,73,88]}$ in the bandgap of $\mathrm{MAPbI}_{3}$ (as shown in Figure 6a,b. We have, however, identified five different defects. Given the low deep defect concentration demonstrated by TEES $\left(<10^{11} \mathrm{~cm}^{-3}\right)$, it is reasonable to consider less stable defects. This is justified considering that DFT cannot model accurately shallow defects using relatively small supercells ${ }^{[48]}$ as in most DFT studies. Also, different DFT calculations found slightly different defect activation energies (defect level to band energy difference). ${ }^{[72-74]}$ 
Table 1. Fingerprints of defects in $\mathrm{MAPbl}_{3}$. Defect parameters of single-crystal $\mathrm{MAPbl}_{3}$ found from the combination of time of flight and Monte Carlo simulation, photo-Hall spectroscopy, and thermoelectric effect spectroscopy.

\begin{tabular}{|c|c|c|c|c|c|c|c|c|}
\hline Trap & Trap type & $\begin{array}{l}\text { Trapping time } \\
{[\mu \mathrm{s}]}\end{array}$ & $\begin{array}{c}\text { Detrappingtime } \\
{[\mu \mathrm{s}]}\end{array}$ & Trap energy & $\begin{array}{l}\text { Electron capture cross- } \\
\text { section }\left[\mathrm{cm}^{2}\right]\end{array}$ & $\begin{array}{l}\text { Hole capture cross- } \\
\text { section }\left[\mathrm{cm}^{2}\right]\end{array}$ & $\begin{array}{c}\text { Concentration } \\
{\left[\mathrm{cm}^{-3}\right]}\end{array}$ & $\begin{array}{l}\text { Assigned defect } \\
\text { nature }^{[72-74]}\end{array}$ \\
\hline$E_{1}$ & Shallow hole trap & 3.5 & 4 & $\begin{array}{c}E_{\mathrm{HI}}=E v+0.3 \mathrm{eV} \\
E_{\mathrm{Tl}}=E v+0.35 \mathrm{eV} \\
\text { a) } E \mathrm{w}_{1} \approx E \mathrm{v}+0.35 \mathrm{eV}\end{array}$ & $2 \times 10^{-11}$ & $\approx 70^{-13}$ & $5.0 \times 10^{10}$ & $\begin{array}{c}(-/ 2-) V_{\mathrm{Pb}} \\
V_{\mathrm{MA}}\end{array}$ \\
\hline$E_{2}$ & Deep hole trap & 60 & $>10^{4}$ & $\begin{array}{c}E_{\mathrm{H} 2}=E v+0.6 \mathrm{eV} \\
E_{\mathrm{T} 2}=E v+0.55 \mathrm{eV} \\
E \mathrm{w}_{2}>E \mathrm{v}+0.56 \mathrm{eV}\end{array}$ & $5 \times 10^{-13}$ & $\approx 10^{-14}$ & $1.1 \times 10^{10}$ & $\begin{array}{c}(0 /-) I_{\mathrm{i}} \\
(0 / 2-) V_{\mathrm{Pb}} \\
(-/ 2-) I_{\mathrm{MA}}\end{array}$ \\
\hline$E_{3}$ & Deep electron trap & 120 & $>10^{4}$ & $\begin{array}{c}E_{\mathrm{H}_{3}}=E \mathrm{c}-0.75 \mathrm{eV} \\
{E \mathrm{w}_{3}}_{3}>E_{\mathrm{C}}-0.7 \mathrm{eV}\end{array}$ & $10^{-14}$ & - & b) $\approx 10^{11}$ & $(0 /+) I_{\mathrm{i}}$ \\
\hline$E_{4}$ & $\begin{array}{c}\text { Shallow electron } \\
\text { trap }\end{array}$ & 100 & 100 & $\begin{array}{l}E_{\mathrm{H}_{4}}<E_{\mathrm{C}}-0.2 \mathrm{eV} \\
{E \mathrm{w}_{4}}_{4} \approx E_{\mathrm{C}}-0.3 \mathrm{eV}\end{array}$ & $\approx 70^{-15}$ & - & $\approx 10^{12}$ & $\begin{array}{r}P b_{\mathrm{i}} \\
M A_{\mathrm{i}}\end{array}$ \\
\hline$E_{5}$ & $\begin{array}{c}\text { Shallow electron } \\
\text { trap }\end{array}$ & 2.5 & 1.5 & 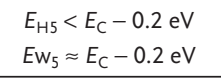 & $\approx 10^{-14}$ & - & $\approx 10^{12}$ & $\begin{array}{c}V_{1} \\
M A_{i}\end{array}$ \\
\hline
\end{tabular}

${ }^{\text {a) }} E_{\mathrm{W} /}$ - energies found by time of flight by fixing their concentration to $10^{11} \mathrm{~cm}^{-3}$; b) Concentrations of electron traps are found using $\mathrm{PHES}$ activation energies.

The shallow hole traps must be negatively (or sometimes neutrally) charged and energetically near the valence band $\left(E_{\mathrm{t}}<0.3 \mathrm{eV}\right)$. We can therefore guess that either negatively charged lead vacancies $V_{\mathrm{Pb}}(-/ 2-)^{[11]}$ or methylammonium vacancies $V_{\text {MA }}$ may be the shallow hole traps (cf. Figure 6 and Table 1 ). Similarly, shallow electron traps $E_{5}$ and $E_{4}$ may be identified with $M A_{\mathrm{i}}$, iodine vacancy $V_{\mathrm{I}}$, and lead interstitial $P b_{\mathrm{i}}{ }^{[72-74]}$ defects.

The deep defects typically have activation energies larger than $0.3 \mathrm{eV} .^{[71]}$ Taking again a step back from the published DFT results, we assign the deep hole trap $E_{2}$ to lead vacancies $(0 / 2-) V_{\mathrm{Pb}}$, substitutional iodine defects in MA site $I_{\mathrm{MA}}(-/ 2-)$, or less probably iodine interstitial $I_{\mathrm{i}}(0 /-)$. This is because the deep electron trap $E_{3}$ may solely be identified as positive iodine interstitial defects $I_{\mathrm{i}}(0 /+)$, which exclude negatively charged iodine defects as hole traps. Several studies ${ }^{[72-74]}$ emphasized deep trap iodine interstitial to be the most probable deep trap in $\mathrm{MAPbI}_{3}$. Considering the energy of the deep trap of $0.55 \mathrm{eV}$ and the low concentration of deep defects, we think that the community would benefit from a more detailed study on $I_{\mathrm{MA}}(-/ 2-)$ and $I_{\mathrm{i}}(+/-)$, which were previously considered to have low capture probability or concentration. ${ }^{\text {[2] }}$ Our results designate that chemical reasoning on defects should also consider the formation of defects in pairs such as $V_{\mathrm{I}}-I_{\mathrm{i}}, V_{\mathrm{Pb}}-P b_{\mathrm{i}}$, and $V_{\mathrm{MA}}-M A_{\mathrm{i}}$ and charge balance. Our results indicate that $I_{\mathrm{i}}, I_{\mathrm{MA}}$, and $V_{\mathrm{Pb}}$ can be the main recombination pathways. Thus the modification of their concentration can improve the stability of MHP devices. On the other hand, secondary phases, ${ }^{[89]}$ defect complexes, solvent (or antisolvent) species, and hydrogen vacancies $V_{\mathrm{H}}{ }^{[90]}$ should be carefully considered as defects in perovskite matrices and have not yet been investigated.

\subsection{Surface Traps against Bulk Traps}

Photoluminescence quantum yield (PLQY) measurement for single crystal samples (Figure S10, Supporting Information) revealed that essential part of free carriers $(P L Q Y \approx 0.01 \%$ ) recombine near the surface region (before $1 \mu \mathrm{s})$. The bulk transport properties probed by ToF (Figures 2c and 3c red curve) demonstrates that much lower charge losses occur in bulk (charge transport efficiency $>50 \%$ ). Such comparison of bulk and surface transport reflects the defective nature of the surface in perovskite. Taking into account PL decay of $\approx 50$ ns (Figure S10, Supporting Information) and capture cross-section of $10^{-14} \mathrm{~cm}^{2}$, the concentration of defects $\approx 10^{13} \mathrm{~cm}^{-3}$ is estimated near the surface (surface defects and bulk defects in the thickness defined by diffusion length). On the other hand, ToF demonstrates higher values of quantum yield (QY) calculated for the surface region in Figure S11, Supporting Information (electron $Q Y \approx 10 \%$ and hole $Q Y \approx 45 \%$ ) because applied voltage bias helps to extract free carriers from the near-surface region. The lower value of electron ToF QY (in comparison with holes) reflects more active electron trapping near the surface region, which is in agreement with their lower diffusion length demonstrated by ToF CWFs and other studies. ${ }^{[1]}$ The free holes diffuse more efficiently in the bulk of the material due to the larger diffusion length. The low recombination velocity $S<500 \mathrm{cms}^{-1}$ obtained from CWFs suggests that part of the surface defects can have a shallow nature.

\section{Effect of Defects on Optoelectronic Devices and Further Pathways of Hybrid Perovskite Development}

This work stresses the need for further theoretical and experimental studies and material processing strategies to control defects in perovskite devices. We expect deep traps to decrease the open-circuit voltage $\left(V_{\mathrm{OC}}\right)$, and the shallow trap to affect the solar cells' saturation current. ${ }^{[92]}$ On the other hand, the low concentration of deep defects (less than $10^{11} \mathrm{~cm}^{-3}$ ) and long diffusion lengths $(34 \mu \mathrm{m})$ suggest that photo-generated carriers can reach electron/hole selective interfaces without any nonradiative losses in 20- $\mu$ m-thick monocrystalline $\mathrm{MAPbI}_{3}$. Our results demonstrate a suppressed interaction of defects and free carriers which explains the boost in stability and efficiency of singly crystal solar cells. ${ }^{[43-93]}$ Further improvements in stability and conversion efficiency can be achieved by controlling 
shallow and deep defects, as demonstrated in this study. Special attention must be given to the collection of free electrons due to the lower electron diffusion length. The slightly n-type doping can be considered to tune electron diffusion length by occupying the electron traps and, as a result, boost solar cell efficiency. The effect should be even stronger in radiation sensors and detectors as they have a thicker thickness and more extensive transit time to interact with traps.

\section{Conclusion}

We performed, for the first time, a multi-technique characterization of the defect properties in $\mathrm{MAPbI}_{3}$ single crystals, which allowed for identifying their density, energy, and capture crosssection and characterizing trapping and detrapping times. We elucidated the effect of each defect on the electron and hole transport, including lifetime and mobility-crucial parameters for MHP device engineering. We found that defects influence both electron (one deep and two shallow traps) and hole (one deep and one shallow trap) transport as indicated with ToF CWF results. We showed that three defects release free carriers after the trapping reducing non-radiative recombination and provide complex charge transport features in $\mathrm{MAPbI}_{3}$. We demonstrated that the shallow traps significantly delay the drift of the free carriers showing for the first time, a fundamental mechanism affecting free charge carrier mobility. In particular, the hole effective mobility decreases from 21 to $10 \mathrm{~cm}^{2} \mathrm{~V}^{-1} \mathrm{~s}^{-1}$, and the electron mobility from 4.0 to $1.7 \mathrm{~cm}^{2} \mathrm{~V}^{-1} \mathrm{~s}^{-1}$. By ToF and $\mathrm{MC}$ simulations we found that holes have a lifetime of 60-200 $\mu$ s and electrons have a lifetime of 20-120 $\mu$ s depending on the crystal quality. This gives hole and electron diffusion lengths of 56-110 and 21-32 $\mu \mathrm{m}$, respectively. The secret of efficient charge transport is active detrapping of carriers by traps preserving charge from recombination as well large diffusion length due to the low concentration of deep defects. The diffusion length of carriers is several times larger than the typical thickness of single crystal solar cells, which allows for collecting more than $99 \%$ of carriers without significant losses in the bulk of the perovskite. We also showed that in the case of radiation sensors and photodetectors' applications, metal contacts collect the dominant part of free carriers before being captured on the traps due to the large lifetime of free carriers.

We used PHES and TEES to reveal the activation energies and relative positions of traps in the bandgap. The electron and hole traps have activation energies of $E \mathrm{v}+0.35, E \mathrm{v}+0.6, E \mathrm{v}+0.75$, $E_{\mathrm{C}}-0.3$, and $E_{\mathrm{C}}-0.2 \mathrm{eV}$, at concentrations below $10^{12} \mathrm{~cm}^{-3}$, and capture cross-sections around $10^{-14} \mathrm{~cm}^{2}$. The combination of ToF and PL reveals a larger traps concentration of $10^{13} \mathrm{~cm}^{-3}$ and more active electron trapping in the near-surface region. In contrast, the free holes diffuse more efficiently in the bulk of the material due to the larger diffusion length. The picture of the bulk and surface charge transport suggest that n-type doping can be an effective strategy to bust electron diffusion length and enhance perovskite devices efficiency. Charge transport characteristics of perovskite material should be further studied at high illumination intensities with an adequate model including several traps due to the presence of several defects and possible asymmetrical trapping of holes and electrons.
Our results will pave the way for defect engineering in halide perovskites. The identification of their energy allows designing architectures where the electron or hole traps are filled, depending on the application. To conclude, a more detailed evaluation of the defect energies and cross-sections is key for the improvement of perovskite stability. The knowledge of the pathways of energy relaxation in the material is necessary to attribute the probability of each degradation pathway. By adequate defect engendering the most critical defect for degradation may be passivated, greatly prolonging the perovskite lifetime.

\section{Experimental Section}

Perovskite Growth: $\mathrm{MAPb}_{3}$ single crystals were grown from a solution of $1 \mathrm{M} \mathrm{MAl}$ and $\mathrm{Pbl}_{2}$ (1:1 mixture, slight excess of MAI) in $\gamma$ butyrolactone mixed at $60{ }^{\circ} \mathrm{C}$. The authors heated the total solution to $90{ }^{\circ} \mathrm{C}$ for $1 \mathrm{~h}$ and then slowly to $110{ }^{\circ} \mathrm{C}$ over $3 \mathrm{~h}$. The solution was then evaporated keeping the solution at $110{ }^{\circ} \mathrm{C}$ to $20 \%$ of its original volume. They collected the crystals before the complete evaporation of the solution. They polished the grown single crystals and evaporated chromium $(\mathrm{Cr})$ contacts for electrical measurements. Samples were encapsulated in non-conductive toluene-based lacquer and stored in a dry chamber. They measured sample resistivity to control possible sample degradation. They observed a constant resistivity of $2 \times 10^{8} \Omega \mathrm{cm}$ before and after all electrical measurements, confirming the negligible effect of illumination and pulsed bias on sampled degradation or decomposition. ${ }^{\text {[94-97] }}$

Surface Treatment: Immediately after sample growth polishing was performed by slow rubbing (moving in circles) the single crystals on a filter paper partially wetted with GBL. The circular motion was performed in a way to make the crystal enter the wet part of the paper and then the dry part in the same movement. Gentle movements were required to avoid the formation of scratches on the crystal surface. After a thorough wash in toluene, the authors polished individual samples on a glass panel with inorganic oil and $\mathrm{AL}_{2} \mathrm{O}_{3}$ suspension until they had a mirror finish. The optical quality of their polishing excluded the presence of defects on the edge of large scratches. As mentioned in the main text, the self-healing properties of the material caused the surface to eliminate surface defects when scratches were below the 1-micrometer size, as indicated by the healing of cracks in Yadavalli et al. ${ }^{[57]}$

Time of Flight Current Spectroscopy: To generate free carriers and control their drift in perovskite samples, the authors used $0.1 \mu \mathrm{s}$ blue laser $(450 \mathrm{~nm})$ and $1 \mathrm{~ms}$ pulse bias. Figure $1 \mathrm{~b}$ demonstrates the main principle of the ToF method. The same setup but with a negative pulse was used to measure electron CWFs. They chose the low illumination intensity of $1 \mu \mathrm{W} \mathrm{cm} \mathrm{cm}^{-2}$ to exclude the noticeable occupation of traps and variation of free carrier lifetime. Such low illumination was used to generate a small carrier density of $10^{6} \mathrm{~cm}^{-3}$. Larger illumination intensities (typically used in PL) produce carrier densities of over $10^{10} \mathrm{~cm}^{-3}$ and fill traps ${ }^{[33]}$ which affected the lifetime of electrons and holes. In addition, a large concentration of photogenerated carriers could deform the electrical field. Due to the low intensity and CWF signal, they used a 200x amplifier to enhance the CWF. The ToF setup had a time resolution of 50 ns. The effect of shallow traps could be detected in the CWF as a long current tail after transit time $\left(T_{R}\right) . T_{R}$ is the time needed to pass by carriers in the semiconductor bulk to reach the opposite electrode. The transit time connects the mobility $(\mu)$ of charge carriers and bias $(V)$ by the relation $T_{R}=L^{2} / \mu \mathrm{V}$. They used different biases to study hole and electron CWF to compensate for the significant difference in carrier mobility. Deep traps typically trap electron and holes without any following detrapping in the time of the experiment $(20 \mathrm{~ms})$. The deeply trapped electron/holes recombined with free holes/electrons captured from the valance/conduction band. The effect of deep traps on CWF was recognized through a single exponential decay which was not affected by steady state concentration of free carries. Note that if the 
lifetime of the free carrier was longer than $T_{R}$, then the carriers never reach the collecting electrode. Adequate bias needed to be applied to investigate the drift of free carriers with different mobilities. The decay dynamics of the current before and after $T_{R}$ stores the information about charge trapping and detrapping events. Trapping time was inversely proportional to the trap concentration $n t_{\mathrm{i}}$ and defect capture crosssection $\sigma_{\mathrm{i}}$. The position of the trap $E_{\mathrm{i}}$ in the bandgap determines the detrapping time. They used the Shockley-Read-Hall recombination mode ${ }^{[98,99]}$ and Shockley-Ramo theorem to describe the dynamics of free charges affected by deep and shallow traps. Because there was no analytical solution of the SHR model with more than a single trap, ${ }^{[48]}$ they simulated the charge transport through Monte Carlo simulation. They found the effect of each trap on the charge transport as well as mobility, lifetime, and rate parameters of the traps (trapping time $\tau_{\mathrm{Ti}}$ and detrapping time $\tau_{\mathrm{Di}}$ ). They measured eight $\mathrm{MAPbl}_{3}$ samples that showed similar hole and electron relaxation dynamics. Among the eight samples, they detected a variation of holes and electron lifetime. In the main text of the paper, they discuss sample A with the largest electron lifetime. The ToF measurements of samples with low electron lifetime (sample B) are given in Figure S4, Supporting Information, for comparison.

Monte Carlo Simulation: Both drift and diffusion of free carriers were included in the MC simulation. ${ }^{[48]}$ The authors included the diffusion process of the charge cloud by means of a normal Gaussian law random offset. Free charge cloud diffused uniformly in all directions, which led to broadening of the transit time region. MC simulation was performed using a ID technique (transverse uniformity of the material was considered) with a total number of particles, $N=10^{5}$. CWFs were calculated using Shockley-Ramo theorem. The MC included the effect of shallow traps and diffusion on the broadening of the drifting charge cloud in space. Without shallow traps, the charge would otherwise have had a Gaussian space distribution equivalent to the hole diffusion length (obtained as the product of hole mobility and lifetime). Charge trapping and detrapping by deep and shallow traps were considered on the basis of the Shockley-Read-Hall generation-recombination model, as shown in Equation S1, Supporting Information. According to the authors' MC simulation model, each charge carrier, whether electron or hole, can have two states: 1) free-drifting carrier; or 2) carrier trapped by one of the traps. MC simulation step changes the state of the MC particle using randomly generated numbers according to probability given by the trapping and detrapping time of a particular trap level. The trapping-detrapping history of each electron and hole can be tracked as demonstrated in Figures $2 \mathrm{~b} d$ and $3 \mathrm{~b}, \mathrm{~d}$. They found parameters (trapping/detrapping time) of each trap in the bandgap by fitting experimental ToF results with MC simulation and least square regression analysis. Effective mobility describes the effect of defects on the delaying of free charge drift. ${ }^{[88]}$ More details on MC can be found in their previous study. ${ }^{[48]}$ In their Monte Carlo simulations (MC) they neglected band-to-band recombination (bimolecular recombination) and Auger recombination due to the low concentration of the photogenerated carriers $\left(\approx 10^{6} \mathrm{~cm}^{-3}\right)$.

Photo-Hall Effect Spectroscopy: The principle of classical Hall-effect measurements and PHES were depicted in Figure $5 \mathrm{~b}$ The longitudinal voltage $V$, current $I$, and the transverse Hall voltage $V_{H}$ were measured directly from the experiment. To calculate the resistivity and Hall mobility, the authors used the average values, including different orientations of the applied current and magnetic field. Measurements were performed at room temperature with a constant magnetic field $B$ of $1 \mathrm{~T}$. The illumination intensity of $1 \mathrm{~mW} \mathrm{~cm} \mathrm{~cm}^{-2}$ was used in the PHES setup.

Thermoelectric Effect Spectroscopy: For the TEES measurements (presented in Figure 5d) the trap levels were filling at $160 \mathrm{~K}$ for $20 \mathrm{~min}$ by illuminating the sample using a white laser source with an intensity of $1 \mathrm{~mW} \mathrm{~cm}{ }^{-2}(\approx 0.01$ sun). Due to absorption near the surface region of $1 \mu \mathrm{m}$, the authors excluded any effect of degradation on TEES signalinduced preferably by bulk. The occupation of traps was a preferable process in TEES experiments; the photodoping process does not contradict the principles of the method. ${ }^{[100-102]}$ The defect concentration they found was not affected by ion diffusion since they do not use bias in TEES. In addition, the concentration could be assessed by ToF from the trapping time, which gives the value for $\approx 10^{10} \mathrm{~cm}^{-3}$ as well. The sample relaxed for $15 \mathrm{~min}$ at $160 \mathrm{~K}$ after illumination. Next, they created the temperature gradient across the sample with cold and hot fingers. The temperature was increased, keeping constant rates in the range $0.5-2.0 \mathrm{~K} \mathrm{~min}^{-1}$. Free carriers detrapped by the traps diffuse in the temperature gradient resulting in current measured by a current meter. Diffused electron and hole produce positive and negative current, respectively, which allowed for distinguishing electron and hole traps. They did not observe shallow trap bands at low temperatures (typically below $250 \mathrm{~K}$ ), probably due to the fast detrapping. Thermal and optical ionization energies were different by $0.05 \mathrm{eV}$, on the edge of the setup resolution $(\approx 0.1 \mathrm{eV}$ for PHES and $\approx 0.15 \mathrm{eV}$ for TEES). Such difference was typically observed in semiconductors and was mainly affected by the nature of a particular defect. ${ }^{[103]}$ Another mechanism responsible for this difference was the effect of phonons on thermal transition probability.

\section{Supporting Information}

Supporting Information is available from the Wiley Online Library or from the author.

\section{Acknowledgements}

A.M., J.P., P.P, M.B., and E.B. thank the Institute of Physics of Charles University for providing the necessary facilities to conduct this research. A.M., J.P., P.P, M.B., and E.B. acknowledge financial support from the Grant Agency of the Czech Republic, Grant No. P102/19/11920S, and the Grant Agency of Charles University, project No. 1234119. H.E. thanks the Ministry of Education, Youth, and Sports of the Czech Republic - National Program of Sustainability (NPU LO1305). The authors gratefully acknowledge Vaclav Dedic, Martin Rejhon, Jan Franc, and Roman Grill for their assistance. A.M. acknowledges financial support from the German Science Foundation (DFG) in the framework of the priority program SPP 2196. D.R.C. thanks the European Union's Horizon 2020 research and innovation programme under the Marie Skłodowoska Curie Grant Agreement No. 893194 and the Sustainability and Energy Research Initiative (SAERI) of the Weizmann Institute for funding, the Minerva Centre for Self-Repairing Systems for Energy \& Sustainability, and the CNRS-Weizmann program for support. The Acknowledgements and reference 91 were updated on November 25, 2021 after initial online publication.

Open access funding enabled and organized by Projekt DEAL.

\section{Conflict of Interest}

The authors declare no conflict of interest.

\section{Data Availability Statement}

The data that support the findings of this study are available from the corresponding author upon reasonable request.

\section{Keywords}

charge transport, defects, halide perovskites, nature of defects, perovskites, perovskites stability, solar cells

Received: May 12, 2021

Revised: August 12, 2021

Published online: September 1, 2021 
[1] T. F. Stocker, Science 2013, 339, 280.

[2] J. Jeong, M. Kim, J. Seo, H. Lu, P. Ahlawat, A. Mishra, Y. Yang, M. A. Hope, F. T. Eickemeyer, M. Kim, Y. J. Yoon, I. W. Choi, B. P. Darwich, S. J. Choi, Y. Jo, J. H. Lee, B. Walker, S. M. Zakeeruddin, L. Emsley, U. Rothlisberger, A. Hagfeldt, D. S. Kim, M. Grätzel, J. Y. Kim, Nature 2021, 592, 381.

[3] M. B. Johansson, L. Xie, B. J. Kim, J. Thyr, T. Kandra, E. M. J. Johansson, M. Göthelid, T. Edvinsson, G. Boschloo, Nano Energy 2020, 78, 105346.

[4] W. Yu, F. Li, L. Yu, M. R. Niazi, Y. Zou, D. Corzo, A. Basu, C. Ma, S. Dey, M. L. Tietze, U. Buttner, X. Wang, Z. Wang, M. N. Hedhili, C. Guo, T. Wu, A. Amassian, Nat. Commun. 2018, 9, 5354.

[5] H. Zhu, A. Liu, Y. Y. Noh, Nat. Electron. 2020, 3, 662.

[6] J. Zhao, L. Zhao, Y. Deng, X. Xiao, Z. Ni, S. Xu, J. Huang, Nat. Photonics 2020, 14, 612.

[7] S. Shrestha, R. Fischer, G. J. Matt, P. Feldner, T. Michel, A. Osvet, I. Levchuk, B. Merle, S. Golkar, H. Chen, S. F. Tedde, O. Schmidt, R. Hock, M. Rührig, M. Göken, W. Heiss, G. Anton, C. J. Brabec, Nat. Photonics 2017, 11, 436.

[8] S. Yakunin, B. M. Benin, Y. Shynkarenko, O. Nazarenko, M. I. Bodnarchuk, D. N. Dirin, C. Hofer, S. Cattaneo, M. V. Kovalenko, Nat. Mater. 2019, 18, 846.

[9] H. Wei, J. Huang, Nat. Commun. 2019, 10, 1066.

[10] Z. Ni, C. Bao, Y. Liu, Q. Jiang, W. Q. Wu, S. Chen, X. Dai, B. Chen B. Hartweg, Z. Yu, Z. Holman, J. Huang, Science 2020, 367, 1352.

[11] D. Meggiolaro, S. G. Motti, E. Mosconi, A. J. Barker, J. Ball, C. A. R. Perini, F. Deschler, A. Petrozza, F. De Angelis, Energy Environ. Sci. 2018, 11, 702.

[12] V. Adinolfi, M. Yuan, R. Comin, E. S. Thibau, D. Shi, M. I. Saidaminov, P. Kanjanaboos, D. Kopilovic, S. Hoogland, Z.-H. Lu, O. M. Bakr, E. H. Sargent, Adv. Mater. 2016, 28, 3406.

[13] M. J. Trimpl, A. D. Wright, K. Schutt, L. R. V. Buizza, Z. Wang, M. B. Johnston, H. J. Snaith, P. Müller-Buschbaum, L. M. Herz, Adv. Funct. Mater. 2020, 30, 2004312.

[14] W. Tress, M. Yavari, K. Domanski, P. Yadav, B. Niesen, J. P. C. Baena, A. Hagfeldt, M. Graetzel, Energy Environ. Sci. 2018 $11,151$.

[15] S. Reichert, Q. An, Y. W. Woo, A. Walsh, Y. Vaynzof, C. Deibel, Nat. Commun. 2020, 11, 6098.

[16] A. Kalam, R. Runjhun, A. Mahapatra, M. M. Tavakoli, S. Trivedi, H. T. Dastjerdi, P. Kumar, J. Lewiński, M. Pandey, D. Prochowicz, P. Yadav, J. Phys. Chem. C 2020, 124, 3496.

[17] G. Gordillo, C. A. Otálora, M. A. Reinoso, J. Appl. Phys. 2017, 122, 075304.

[18] A. Baumann, S. Väth, P. Rieder, M. C. Heiber, K. Tvingstedt, V. Dyakonov, J. Phys. Chem. Lett. 2015, 6, 2350.

[19] G. Landi, H. C. Neitzert, C. Barone, C. Mauro, F. Lang, S. Albrecht, B. Rech, S. Pagano, Adv. Sci. 2017, 4, 1700183.

[20] Y. Chen, H. T. Yi, V. Podzorov, Phys. Rev. Appl. 2016, 5, 034008.

[21] D. Shi, V. Adinolfi, R. Comin, M. Yuan, E. Alarousu, A. Buin, Y. Chen, S. Hoogland, A. Rothenberger, K. Katsiev, Y. Losovyj, X. Zhang, P. A. Dowben, O. F. Mohammed, E. H. Sargent, O. M. Bakr, Science 2015, 347, 519.

[22] Y. Bi, E. M. Hutter, Y. Fang, Q. Dong, J. Huang, T. J. Savenije, J. Phys. Chem. Lett. 2016, 7, 923.

[23] A. Bercegol, F. J. Ramos, A. Rebai, T. Guillemot, D. Ory, J. Rousset, L. Lombez, J. Phys. Chem. C 2018, 122, 24570.

[24] Y. Chen, H. T. Yi, X. Wu, R. Haroldson, Y. N. Gartstein, Y. I. Rodionov, K. S. Tikhonov, A. Zakhidov, X. Y. Zhu, V. Podzorov, Nat. Commun. 2016, 7, 12253.

[25] J. Euvrard, O. Gunawan, D. B. Mitzi, Adv. Energy Mater. 2019, 9, 1902706.

[26] D. W. de Quilettes, S. M. Vorpahl, S. D. Stranks, H. Nagaoka, G. E. Eperon, M. E. Ziffer, H. J. Snaith, D. S. Ginger, Science 2015 348,683 .
[27] D. W. deQuilettes, S. Koch, S. Burke, R. K. Paranji, A. J. Shropshire, M. E. Ziffer, D. S. Ginger, ACS Energy Lett. 2016, 1, 438.

[28] A. Al-Ashouri, E. Köhnen, B. Li, A. Magomedov, H. Hempel, P. Caprioglio, J. A. Márquez, A. B. Morales Vilches, E. Kasparavicius, J. A. Smith, N. Phung, D. Menzel, M. Grischek, L. Kegelmann, D. Skroblin, C. Gollwitzer, T. Malinauskas, M. Jošt, G. Matič, B. Rech, R. Schlatmann, M. Topič, L. Korte, A. Abate, B. Stannowski, D. Neher, M. Stolterfoht, T. Unold, V. Getautis, S. Albrecht, Science 2020, 370, 1300.

[29] O. Gunawan, S. R. Pae, D. M. Bishop, Y. Virgus, J. H. Noh, N. J. Jeon, Y. S. Lee, X. Shao, T. Todorov, D. B. Mitzi, B. Shin, Nature 2019, 575, 151.

[30] F. Zhang, B. Yang, Y. Li, W. Deng, R. He, J. Mater. Chem. C 2017, $5,8431$.

[31] Q. Dong, Y. Fang, Y. Shao, P. Mulligan, J. Qiu, L. Cao, J. Huang, Science 2015, 347, 967.

[32] G. A. Elbaz, D. B. Straus, O. E. Semonin, T. D. Hull, D. W. Paley, P. Kim, J. S. Owen, C. R. Kagan, X. Roy, Nano Lett. 2017, 17, 1727.

[33] I. Levine, S. Gupta, A. Bera, D. Ceratti, G. Hodes, D. Cahen, D. Guo, T. J. Savenije, J. Ávila, H. J. Bolink, O. Millo, D. Azulay, I. Balberg, J. Appl. Phys. 2018, 124, 103103.

[34] W. Kong, Z. Ye, Z. Qi, B. Zhang, M. Wang, A. Rahimi-Iman, H. Wu, Phys. Chem. Chem. Phys. 2015, 17, 16405.

[35] M. Keshavarz, M. Ottesen, S. Wiedmann, M. Wharmby, R. Küchler, H. Yuan, E. Debroye, J. A. Steele, J. Martens, N. E. Hussey, M. Bremholm, M. B. J. Roeffaers, J. Hofkens, Adv. Mater. 2019, 37 1900521

[36] P. S. Whitfield, N. Herron, W. E. Guise, K. Page, Y. Q. Cheng, I. Milas, M. K. Crawford, Sci. Rep. 2016, 6, 35685

[37] E. V. Peán, S. Dimitrov, C. S. De Castro, M. L. Davies, Phys. Chem. Chem. Phys. 2020, 22, 28345.

[38] J. C. de Mello, H. F. Wittmann, R. H. Friend, Adv. Mater. 1997, 9, 230 .

[39] M. Stolterfoht, C. M. Wolff, J. A. Márquez, S. Zhang, C. J. Hages, D. Rothhardt, S. Albrecht, P. L. Burn, P. Meredith, T. Unold, D. Neher, Nat. Energy 2018, 3, 847.

[40] T. Kirchartz, J. A. Márquez, M. Stolterfoht, T. Unold, Adv. Energy Mater. 2020, 10, 1904134.

[41] Y. Cheng, X. Liu, Z. Guan, M. Li, Z. Zeng, H. Li, S. Tsang, A. G. Aberle, F. Lin, Adv. Mater. 2021, 33, 2006170.

[42] Y. Song, W. Bi, A. Wang, X. Liu, Y. Kang, Q. Dong, Nat. Commun. 2020, 11, 274

[43] Y. Lei, Y. Chen, R. Zhang, Y. Li, Q. Yan, S. Lee, Y. Yu, H. Tsai, W. Choi, K. Wang, Y. Luo, Y. Gu, X. Zheng, C. Wang, C. Wang, H. Hu, Y. Li, B. Qi, M. Lin, Z. Zhang, S. A. Dayeh, M. Pharr, D. P. Fenning, Y. H. Lo, J. Luo, K. Yang, J. Yoo, W. Nie, S. Xu, Nature 2020, 583, 790.

[44] Y. Yang, Y. Yan, M. Yang, S. Choi, K. Zhu, J. M. Luther, M. C. Beard, Nat. Commun. 2015, 6, 1.

[45] Z. Chen, B. Turedi, A. Y. Alsalloum, C. Yang, X. Zheng, I. Gereige, A. Alsaggaf, O. F. Mohammed, O. M. Bakr, ACS Energy Lett. 2019, 4, 1258.

[46] B. Turedi, V. Yeddu, X. Zheng, D. Y. Kim, O. M. Bakr, M. I. Saidaminov, ACS Energy Lett. 2021, 6, 631.

[47] Y. Liu, A. V. levlev, L. Collins, A. Belianinov, J. K. Keum, M. Ahmadi, S. Jesse, S. T. Retterer, K. Xiao, J. Huang, B. G. Sumpter, S. V. Kalinin, B. Hu, O. S. Ovchinnikova, Adv. Electron. Mater. 2020, 6, 1901235.

[48] A. Musiienko, J. Pipek, P. Praus, M. Brynza, E. Belas, B. Dryzhakov, M.-H. Du, M. Ahmadi, R. Grill, Sci. Adv. 2020, 6, eabb6393.

[49] G. Hodes, P. V. Kamat, J. Phys. Chem. Lett. 2015, 6, 4090.

[50] L. M. Herz, ACS Energy Lett. 2017, 2, 1539.

[51] A. I. Pointon, N. E. Grant, S. L. Pain, J. T. White, J. D. Murphy, Appl. Phys. Lett. 2020, 116, 121601. 
[52] D. R. Ceratti, Y. Rakita, L. Cremonesi, R. Tenne, V. Kalchenko, M. Elbaum, D. Oron, M. A. C. Potenza, G. Hodes, D. Cahen, Adv. Mater. 2018, 30, 1706273.

[53] D. R. Ceratti, A. V. Cohen, R. Tenne, Y. Rakita, L. Snarski, N. P. Jasti, L. Cremonesi, R. Cohen, M. Weitman, I. Rosenhek-Goldian, I. Kaplan-Ashiri, T. Bendikov, V. Kalchenko, M. Elbaum, M. A. C. Potenza, L. Kronik, G. Hodes, D. Cahen, Mater. Horiz. 2021, 8, 1570

[54] Y. Yu, F. Zhang, H. Yu, Sol. Energy 2020, 209, 408.

[55] S. Jariwala, S. Burke, S. Dunfield, R. C. Shallcross, M. Taddei, J. Wang, G. E. Eperon, N. R. Armstrong, J. J. Berry, D. S. Ginger, Chem. Mater. 2021, 33, 5035.

[56] Q. Chen, H. Zhou, T. Bin Song, S. Luo, Z. Hong, H. S. Duan, L. Dou, Y. Liu, Y. Yang, Nano Lett. 2014, 14, 4158.

[57] S. K. Yadavalli, Z. Dai, H. Zhou, Y. Zhou, N. P. Padture, Acta Mater. 2020, 187, 112.

[58] B. Guzelturk, T. Winkler, T. W. J. Van de Goor, M. D. Smith, S. A. Bourelle, S. Feldmann, M. Trigo, S. W. Teitelbaum, H. G. Steinrück, G. A. de la Pena, R. Alonso-Mori, D. Zhu, T. Sato, H. I. Karunadasa, M. F. Toney, F. Deschler, A. M. Lindenberg, Nat. Mater. 2021, 20, 618.

[59] G. Xing, N. Mathews, S. Sun, S. S. Lim, Y. M. Lam, M. Grä̈zel, S. Mhaisalkar, T. C. Sum, Science 2013, 342, 344.

[60] O. Baussens, L. Maturana, S. Amari, J. Zaccaro, J.-M. Verilhac, L. Hirsch, E. Gros-Daillon, Appl. Phys. Lett. 2020, 117, 041904.

[61] R. L. Milot, G. E. Eperon, H. J. Snaith, M. B. Johnston, L. M. Herz, Adv. Funct. Mater. 2015, 25, 6218.

[62] O. G. Reid, M. Yang, N. Kopidakis, K. Zhu, G. Rumbles, ACS Energy Lett. 2016, 1, 561.

[63] E. M. Hutter, G. E. Eperon, S. D. Stranks, T. J. Savenije, J. Phys. Chem. Lett. 2015, 6, 3082.

[64] C. S. Ponseca, T. J. Savenije, M. Abdellah, K. Zheng, A. Yartsev, T. Pascher, T. Harlang, P. Chabera, T. Pullerits, A. Stepanov, J. P. Wolf, V. Sundström, J. Am. Chem. Soc. 2014, 136, 5189.

[65] Y. Liu, Z. Yang, S. F. Liu, Adv. Sci. 2018, 5, 1700471.

[66] H. Wei, D. DeSantis, W. Wei, Y. Deng, D. Guo, T. J. Savenije, L. Cao, J. Huang, Nat. Mater. 2017, 16, 826.

[67] V. Bruevich, H. H. Choi, V. Podzorov, Adv. Funct. Mater. 2021, 31, 2006178.

[68] N. T. P. Hartono, S. Sun, M. C. Gélvez-Rueda, P. J. Pierone, M. P. Erodici, J. Yoo, F. Wei, M. Bawendi, F. C. Grozema, M. J. Sher, T. Buonassisi, J. P. Correa-Baena, J. Mater. Chem. A 2019, 7, 23949.

[69] J. Lim, M. T. Hörantner, N. Sakai, J. M. Ball, S. Mahesh, N. K. Noel, Y. H. Lin, J. B. Patel, D. P. McMeekin, M. B. Johnston, B. Wenger, H. J. Snaith, Energy Environ. Sci. 2019, 12, 169.

[70] T. Takebe, J. Saraie, H. Matsunami, J. Appl. Phys. 1982, 53, 457.

[71] A. Musiienko, P. Moravec, R. Grill, P. Praus, I. Vasylchenko, J. Pekarek, J. Tisdale, K. Ridzonova, E. Belas, L. Landová, B. Hu, E. Lukosi, M. Ahmadi, Energy Environ. Sci. 2019, 12, 1413.

[72] X. Zhang, M. E. Turiansky, J. X. Shen, C. G. Van De Walle, Phys. Rev. B 2020, 101, 140101.

[73] W. J. Yin, T. Shi, Y. Yan, Appl. Phys. Lett. 2014, 104, 063903.

[74] D. Meggiolaro, F. De Angelis, ACS Energy Lett. 2018, 3, 2206.

[75] J. Dhar, S. Sil, A. Dey, P. P. Ray, D. Sanyal, J. Phys. Chem. Lett. 2017, 8, 1745.

[76] A. Musiienko, R. Grill, J. Pekárek, E. Belas, P. Praus, J. Pipek, V. Dědič, H. Elhadidy, Appl. Phys. Lett. 2017, 111, 082103.

[77] S. Ravishankar, T. Unold, T. Kirchartz, Science 2021, 371, eabd8014.
[78] H. Jin, E. Debroye, M. Keshavarz, I. G. Scheblykin, M. B. J. Roeffaers, J. Hofkens, J. A. Steele, Mater. Horiz. 2020, 7, 397.

[79] H.-H. Fang, S. Adjokatse, H. Wei, J. Yang, G. R. Blake, J. Huang, J. Even, M. A. Loi, Sci. Adv. 2016, 2, el600534.

[80] Z.-Y. Zhang, H.-Y. Wang, Y.-X. Zhang, Y.-W. Hao, C. Sun, Y. Zhang, B.-R. Gao, Q.-D. Chen, H.-B. Sun, Sci. Rep. 2016, 6, 27286.

[81] Q. Dong, J. Mendes, L. Lei, D. Seyitliyev, L. Zhu, S. He, K. Gundogdu, F. So, ACS Appl. Mater. Interfaces 2020, 12, 48845.

[82] M. H. Futscher, J. M. Lee, L. McGovern, L. A. Muscarella, T. Wang, M. I. Haider, A. Fakharuddin, L. Schmidt-Mende, B. Ehrler, Mater. Horiz. 2019, 6, 1497.

[83] P. Calado, A. M. Telford, D. Bryant, X. Li, J. Nelson, B. C. O’Regan, P. R. F. Barnes, Nat. Commun. 2016, 7, 13831.

[84] G. Xia, B. Huang, Y. Zhang, X. Zhao, C. Wang, C. Jia, J. Zhao, W. Chen, J. Li, Adv. Mater. 2019, 31, 1902870.

[85] W. Zhou, J. Gu, Z. Yang, M. Wang, Q. Zhao, J. Phys. D: Appl. Phys. 2020, 54, 063001.

[86] D. Lan, Prog. Photovoltaics Res. Appl. 2020, 28, 533.

[87] E. A. Duijnstee, J. M. Ball, V. M. Le Corre, L. J. A. Koster, H. J. Snaith, J. Lim, ACS Energy Lett. 2020, 5, 376.

[88] J. Euvrard, Y. Yan, D. B. Mitzi, Nat. Rev. Mater. 2021, 6, 531

[89] C. C. Stoumpos, C. D. Malliakas, M. G. Kanatzidis, Inorg. Chem. 2013, 52, 9019.

[90] D. R. Ceratti, A. Zohar, R. Kozlov, H. Dong, G. Uraltsev, O. Girshevitz, I. Pinkas, L. Avram, G. Hodes, D. Cahen, Adv. Mater. 2020, 32, 2002467.

[91] I. Levine, A. Al-Ashouri, A. Musiienko, H. Hempel, A. Magomedov, A. Drevilkauskaite, V. Getautis, D. Menzel, K. Hinrichs, T. Unold, S. Albrecht, T. Dittrich, Joule 2021, ISSN 2542-4351, https://doi.org/10.1016/j.joule.2021.07.016.

[92] D. Pal, S. Das, Opt. Mater. 2020, 108, 110453.

[93] A. Y. Alsalloum, B. Turedi, K. Almasabi, X. Zheng, R. Naphade, S. D. Stranks, O. F. Mohammed, O. M. Bakr, Energy Environ. Sci. 2021, 14, 2263.

[94] C. Bao, J. Yang, W. Zhu, X. Zhou, H. Gao, F. Li, G. Fu, T. Yu, Z. Zou, Chem. Commun. 2015, 51, 15426

[95] T. Liu, X. Zhao, J. Li, Z. Liu, F. Liscio, S. Milita, B. C. Schroeder, O. Fenwick, Nat. Commun. 2019, 10, 5750

[96] C. L. Watts, L. Aspitarte, Y. H. Lin, W. Li, R. Elzein, R. Addou, M. J. Hong, G. S. Herman, H. J. Snaith, J. G. Labram, Commun. Phys. 2020, 3, 73.

[97] Y. Ren, H. Ma, W. Wang, Z. Wang, H. Xu, X. Zhao, W. Liu, J. Ma, Y. Liu, Adv. Mater. Technol. 2019, 4, 1800238.

[98] A. Musiienko, R. Grill, P. Moravec, G. Korcsmáros, M. Rejhon, J. Pekárek, H. Elhadidy, L. Šedivý, I. Vasylchenko, J. Appl. Phys. 2018, 123, 161502

[99] A. Musiienko, R. Grill, P. Moravec, P. Fochuk, I. Vasylchenko, H. Elhadidy, L. Šedivý, Phys. Rev. Appl. 2018, 10, 014019.

[100] S. Feldmann, S. Macpherson, S. P. Senanayak, M. Abdi-Jalebi, J. P. H. Rivett, G. Nan, G. D. Tainter, T. A. S. Doherty, K. Frohna, E. Ringe, R. H. Friend, H. Sirringhaus, M. Saliba, D. Beljonne, S. D. Stranks, F. Deschler, Nat. Photonics 2019, 14, 123.

[101] S. D. Stranks, V. M. Burlakov, T. Leijtens, J. M. Ball, A. Goriely, H. J. Snaith, Phys. Rev. Appl. 2014, 2, 034007.

[102] A. Kiligaridis, P. A. Frantsuzov, A. Yangui, S. Seth, J. Li, Q. An, Y. Vaynzof, I. G. Scheblykin, Nat. Commun. 2021, 12, 3329.

[103] D. Wickramaratne, J. X. Shen, C. E. Dreyer, A. Alkauskas, C. G. Van de Walle, Phys. Rev. B 2019, 99, 205202. 